\title{
A Short and Efficient Route to the \\ Fully Functionalized Polar Core of Scyphostatin
}

\author{
Emmanuel N. Pitsinos, ${ }^{*}$ and Ana Cruz
}

SUPPORTING INFORMATION 
General: All reactions were carried out under a dry argon atmosphere with anhydrous, freshly distilled solvents under anhydrous conditions unless otherwise noted. All reactions were magnetically stirred with Teflon stir bars, and temperatures were measured externally. Reactions requiring anhydrous conditions were carried out in oven dried $\left(120^{\circ} \mathrm{C}, 24 \mathrm{~h}\right)$ or flame dried (vacuum $<0.5$ Torr) glassware. Yields refer to chromatographically and spectroscopically ( ${ }^{1} \mathrm{H}$ NMR) homogeneous materials. All reagents were obtained from Aldrich Chemical Co. Inc. and used without further purification. All reactions were monitored by thin layer chromatography (TLC) carried out on $0.25-\mathrm{mm}$ E.Merck silica gel plates (60F-254). E.Merck silica gel (60, particle size $0.040-0.063 \mathrm{~mm}$ ) was used for flash column chromatography. Nuclear magnetic resonance (NMR) spectra were recorded on a Bruker AM250 or a Bruker Advance DRX-500 instrument as noted individually. Chemical shifts are measured in parts per million $(\delta)$ relative to the deuterated solvent used in the experiment. Multiplicities are designated as singlet (s), doublet $(\mathrm{d})$, triplet $(\mathrm{t})$, or multiplet $(\mathrm{m})$. Broad or obscured peaks are indicated as "br" or "obs" respectively. 


\section{7-Benzyloxy-3-hexadecanoylamino-3,4-dihydro-2H-1-benzopyran (18).}

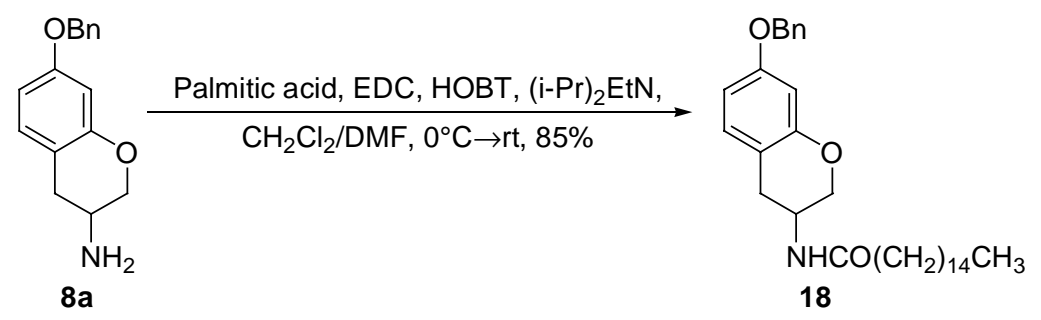

To a stirred solution of 7-benzyloxy-3-amino-3,4-dihydro-2H-1-benzopyran (8a) $(0.51 \mathrm{~g}, 2.0 \mathrm{mmol})$ and palmitic acid $(0.54 \mathrm{~g}, 2.1 \mathrm{mmol})$ in a mixture of dichloromethane $(10 \mathrm{~mL})$ and DMF $(1.5 \mathrm{~mL})$ were added at $0{ }^{\circ} \mathrm{C}$ HOBT $(17 \mathrm{mg}, 0.13 \mathrm{mmol}), \mathrm{N}, \mathrm{N}$-diisopropylethylamine $(0.37 \mathrm{~mL}, 2.1 \mathrm{mmol})$ and $\operatorname{EDC}(0.40 \mathrm{~g}, 2.1 \mathrm{mmol})$. The mixture was stirred for $2 \mathrm{~h}$ at $0{ }^{\circ} \mathrm{C}$ and then for $20 \mathrm{~h}$ at ambient temperature. Upon reaction completion, the mixture was poured in a saturated aqueous solution of ammonium chloride $(30 \mathrm{~mL})$ and extracted with dichloromethane $(3 \times 10 \mathrm{~mL})$. The combined organic phases were washed sequentially with water $(3 \times 10 \mathrm{~mL})$ and brine $(10 \mathrm{~mL})$, dried over $\mathrm{Na}_{2} \mathrm{SO}_{4}$ and concentrated under reduced pressure. The residue thus obtained was purified by flash column chromatography (8:2 hexane-ethyl acetate) to produce 7-benzyloxy-3-hexadecanoylamino-3,4-dihydro-2H-1benzopyran (18) $(0.84 \mathrm{~g}, 85 \%)$ as amorphous white solid: $R_{\mathrm{f}} 0.34$ (8:2 hexane-ethyl acetate); ${ }^{1} \mathrm{H}$ NMR $\left(250 \mathrm{MHz}, \mathrm{CDCl}_{3}\right) \delta 7.44-7.30(\mathrm{~m}, 5 \mathrm{H}, \mathrm{PhH})$, $6.94(\mathrm{~d}, J=8.4 \mathrm{~Hz}, 1 \mathrm{H}, \operatorname{ArH}), 6.58(\mathrm{dd}, J=8.4,2.5 \mathrm{~Hz}, 1 \mathrm{H}, \operatorname{ArH}), 6.48(\mathrm{~d}, J$ $=2.5 \mathrm{~Hz}, 1 \mathrm{H}, \operatorname{ArH}), 5.75$ (brd, $J=7.7 \mathrm{~Hz}, 1 \mathrm{H}, \mathrm{NHCO}), 5.02(\mathrm{~s}, 2 \mathrm{H}$, $\mathrm{OCH}_{2} \mathrm{Ph}$ ), 4.54-4.43 (m, $1 \mathrm{H}, \mathrm{CHNHCO}$ ), 4.10 (ddd, $J=10.8,2.9,2.2 \mathrm{~Hz}, 1$ $\mathrm{H}, \mathrm{CHHO}$ ), 4.06 (dd, $J=10.8,1.1 \mathrm{~Hz}, 1 \mathrm{H}, \mathrm{CHHO}$ ), 3.05 (dd, $J=16.5,5.2 \mathrm{~Hz}$, $1 \mathrm{H}, \mathrm{CHHCH}$ ), 2.66 (brd $J=16.5 \mathrm{~Hz}, 1 \mathrm{H}, \mathrm{CH} H \mathrm{CH}$ ), $2.13(\mathrm{t}, J=7.6 \mathrm{~Hz}, 2 \mathrm{H}$, $\mathrm{COCH}_{2}$ ), 1.67-1.53 (m, $\left.2 \mathrm{H}, \mathrm{COCH}_{2} \mathrm{CH}_{2}\right), 1.25$ (brs, $\left.24 \mathrm{H},\left(\mathrm{CH}_{2}\right)_{12}\right), 0.88$ (t, $J$ $\left.=6.5 \mathrm{~Hz}, 3 \mathrm{H}, \mathrm{CH}_{3}\right) ;{ }^{13} \mathrm{C} \mathrm{NMR}\left(62.5 \mathrm{MHz}, \mathrm{CDCl}_{3}\right) \delta 173.0,158.5,154.5$, $136.9,130.9,128.6,127.9,127.4,111.5,108.9,102.7,70.0,68.2,42.1,36.7$, 31.9, 29.6, 29.4, 29.3, 25.6, 22.7, 14.1; HR-ESI: $\mathrm{m} / \mathrm{z}: 494.3636,\left[\mathrm{M}^{+} \mathrm{H}^{+}\right]$for the compound $\mathrm{C}_{32} \mathrm{H}_{47} \mathrm{NO}_{3}$ requires 494.3629 . 
7-Hydroxy-3-hexadecanoylamino-3,4-dihydro-2H-1-benzopyran (5).
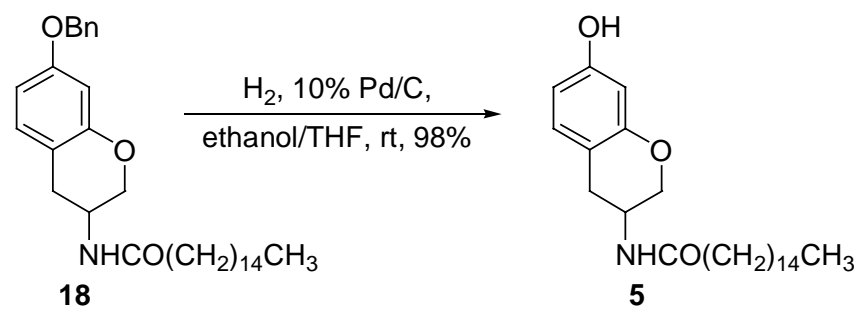

A solution of 7-benzyloxy-3-hexadecanoylamino-3,4-dihydro-2H-1-benzopyran (18) $(1.2 \mathrm{~g}, 2.4 \mathrm{mmol})$ in a mixture of ethanol $(100 \mathrm{~mL})$ and THF $(200 \mathrm{~mL})$ was subjected to hydrogenolysis over $10 \% \mathrm{Pd} / \mathrm{C}$ at ambient temperature. After $12 \mathrm{~h}$, filtration of the reaction mixture through Celite $^{\circledR}$ and removal of the solvent under reduced pressure provided phenol $5(0.96 \mathrm{mg}, 98 \%)$ as an amorphous white solid: $R_{\mathrm{f}} 0.14$ (3:97 methanol-dichloromethane); ${ }^{1} \mathrm{H}$ NMR (250 MHz, 1:1 CDCl $:$ :DMSO-d 6 ) $\delta 8.85$ (brs, $1 \mathrm{H}, \operatorname{ArOH}), 7.46(\mathrm{~d}, J=7.3 \mathrm{~Hz}$, $1 \mathrm{H}, \mathrm{NHCO}), 6.73(\mathrm{~d}, J=8.2 \mathrm{~Hz}, 1 \mathrm{H}, \operatorname{ArH}), 6.27(\mathrm{dd}, J=8.2,2.3 \mathrm{~Hz}, 1 \mathrm{H}$, $\operatorname{ArH}), 6.17(\mathrm{~d}, J=2.3 \mathrm{~Hz}, 1 \mathrm{H}, \mathrm{ArH}), 4.20-4.09(\mathrm{~m}, 1 \mathrm{H}, \mathrm{CHNHCO}), 4.00$ (dd, $J=10.4,2.1 \mathrm{~Hz}, 1 \mathrm{H}, \mathrm{CHHO}$ ), 3.76 (dd, $J=10.4,7.0 \mathrm{~Hz}, 1 \mathrm{H}, \mathrm{CH} H \mathrm{O}$ ), 2.82 (dd, $J=15.9,5.5 \mathrm{~Hz}, 1 \mathrm{H}, \mathrm{CHHCH}$ ), $2.55(\mathrm{dd} J=15.9,7.1 \mathrm{~Hz}, 1 \mathrm{H}, \mathrm{CH} H C H$ ), 2.07 (t, $J=7.5 \mathrm{~Hz}, 2 \mathrm{H}, \mathrm{COCH}_{2}$ ), 1.54-1.44 (m, $2 \mathrm{H}, \mathrm{COCH}_{2} \mathrm{CH}_{2}$ ), 1.18 (brs, $\left.24 \mathrm{H},\left(\mathrm{CH}_{2}\right)_{12}\right), 0.81\left(\mathrm{t}, \mathrm{J}=6.5 \mathrm{~Hz}, 3 \mathrm{H}, \mathrm{CH}_{3}\right) ;{ }^{13} \mathrm{C} \mathrm{NMR}(62.5 \mathrm{MHz}, 1: 1$ $\left.\mathrm{CDCl}_{3}: \mathrm{DMSO}_{-} \mathrm{d}_{6}\right) \delta 172.5,156.2,153.9,129.7,109.9,108.1,102.4,67.1$, 41.8, 35.3, 31.1, 29.4, 28.8, 28.7, 28.6, 28.5, 25.0, 21.8, 13.5; HR-ESI: m/z: 426.2973, $\left[\mathrm{M}+\mathrm{Na}^{+}\right]$for the compound $\mathrm{C}_{25} \mathrm{H}_{41} \mathrm{NO}_{3}$ requires 426.2979 .

\section{Preparation of para-quinols $9 a$ and $9 b$.}
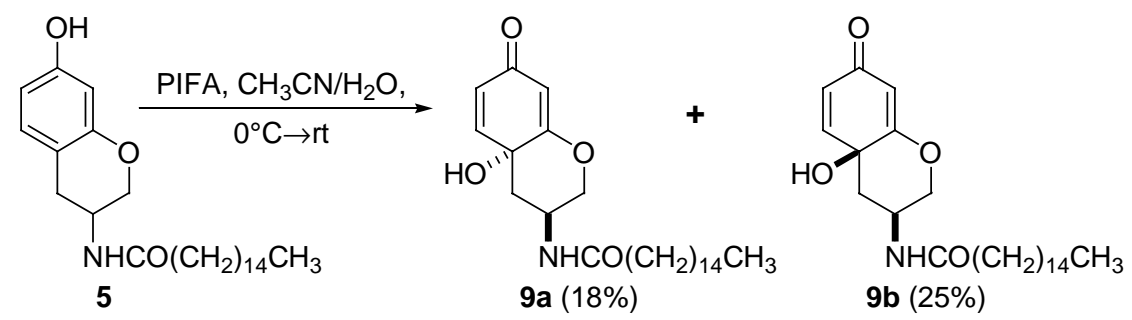

To a vigorously stirred fine suspension of phenol 5 (100 $\mathrm{mg}, 0.25 \mathrm{mmol})$ in a mixture of acetonitrile $(20 \mathrm{~mL})$ and water $(1 \mathrm{~mL})$ was added at $0{ }^{\circ} \mathrm{C}$, [bis(trifluoroacetoxy)iodo]benzene $(200 \mathrm{mg}, 0.47 \mathrm{mmol})$. The mixture was 
stirred for $1 \mathrm{~h}$ at $0{ }^{\circ} \mathrm{C}$ and then at ambient temperature for $6 \mathrm{~h}$. The reaction mixture was then poured in water $(40 \mathrm{~mL})$ and extracted with dichloromethane $(4 \times 15 \mathrm{~mL})$. The combined organic phases were washed sequentially with water $(3 \times 10 \mathrm{~mL})$ and brine $(10 \mathrm{~mL})$, dried over $\mathrm{Na}_{2} \mathrm{SO}_{4}$ and concentrated under reduced pressure. The residue thus obtained was purified by flash column chromatography (3:97 methanol-dichloromethane) to produce, in order of elution,

compound $9 \mathrm{~b}$ as light yellow foam $(25.7 \mathrm{mg}, 25 \%)$ : ${ }^{1} \mathrm{H}$ NMR $(500 \mathrm{MHz}$, $\left.\mathrm{CDCl}_{3}\right) \delta 7.02$ (brd, $J=8.0 \mathrm{~Hz}, 1 \mathrm{H}, \mathrm{NHCO}$ ), 6.61 (d, $J=9.7 \mathrm{~Hz}, 1 \mathrm{H}, \mathrm{CH}=$ ), $6.12(\mathrm{~d}, \mathrm{~J}=9.7 \mathrm{~Hz}, 1 \mathrm{H}, \mathrm{CH}=), 5.77(\mathrm{~s}, 1 \mathrm{H}, \mathrm{CH}=), 4.46-4.39(\mathrm{~m}, 2 \mathrm{H}$, $\mathrm{CHNH}+\mathrm{CHHO}$ ), 4.05 (dd, $J=11.2,3.8 \mathrm{~Hz}, 1 \mathrm{H}, \mathrm{CHHO}$ ), 3.61 (brs, $1 \mathrm{H}, \mathrm{OH}$ ), $2.22\left(\mathrm{t}, J=7.5 \mathrm{~Hz}, 2 \mathrm{H}, \mathrm{COCH} \mathrm{H}_{2}\right.$ ), $2.17(\mathrm{dd}, J=12.9,3.0 \mathrm{~Hz}, 1 \mathrm{H}, \mathrm{CHHCHNH}$ ), 2.05 (dd, $J=12.9,6.0 \mathrm{~Hz}, 1 \mathrm{H}, \mathrm{CH} H \mathrm{CHNH}), 1.67-1.61\left(\mathrm{~m}, 2 \mathrm{H}, \mathrm{COCH}_{2} \mathrm{CH}_{2}\right.$ ), 1.35-1.21 (m, $\left.24 \mathrm{H},\left(\mathrm{CH}_{2}\right){ }_{12} \mathrm{CH}_{3}\right), 0.88\left(\mathrm{t}, J=6.7 \mathrm{~Hz}, 3 \mathrm{H}, \mathrm{CH}_{2} \mathrm{CH}_{3}\right) ;{ }^{13} \mathrm{C} \mathrm{NMR}$ $\left(62.5 \mathrm{MHz}, \mathrm{CDCl}_{3}\right) \delta 187.8,172.9,172.2,145.4,127.9,109.7,73.1,65.6$, $42.4,36.9,35.9,31.9,29.6,29.5,29.3,29.2,25.6,22.7,14.1$; HR-MALDIFTMS: $m / z: 420.3112,\left[\mathrm{M}^{+} \mathrm{H}^{+}\right]$for the compound $\mathrm{C}_{25} \mathrm{H}_{41} \mathrm{NO}_{4}$ requires 420.3108 .

and compound 9a as light yellow foam (18.7 mg, $18 \%)$ : ${ }^{1} \mathrm{H}$ NMR $(500 \mathrm{MHz}$, $\left.\mathrm{CDCl}_{3}\right) \delta 6.60(\mathrm{~d}, J=9.7 \mathrm{~Hz}, 1 \mathrm{H}, \mathrm{CH}=), 6.04(\mathrm{~d}, J=9.7 \mathrm{~Hz}, 1 \mathrm{H}, \mathrm{CH}=), 5.90$ (brd, $J=7.5 \mathrm{~Hz}, 1 \mathrm{H}, \mathrm{NHCO}$ ), 5.66 (s, $1 \mathrm{H}, \mathrm{CH}=$ ), 4.91 (dd, $J=10.9,2.5 \mathrm{~Hz}, 1$ $\mathrm{H}, \mathrm{CHHO}), 4.62-4.56(\mathrm{~m}, 1 \mathrm{H}, \mathrm{CHNH}), 4.01(\mathrm{dd}, J=10.9,2.7 \mathrm{~Hz}, 1 \mathrm{H}$, CHHO), 3.06 (brs, $1 \mathrm{H}, \mathrm{OH}$ ), 2.67 (dd, $J=14.6,8.3 \mathrm{~Hz}, 1 \mathrm{H}, \mathrm{CHHCHNH}$ ), $2.15\left(\mathrm{t}, J=7.4 \mathrm{~Hz}, 2 \mathrm{H}, \quad \mathrm{COCH}_{2}\right), \quad 1.70-1.55(\mathrm{~m}, 3 \mathrm{H}$, $\mathrm{COCH}_{2} \mathrm{CH}_{2}+\mathrm{CH} H \mathrm{CHNH}$ ), 1.24 (brs, $\left.24 \mathrm{H},\left(\mathrm{CH}_{2}\right)_{12} \mathrm{CH}_{3}\right), 0.87$ (t, $J=6.6 \mathrm{~Hz}, 3$ $\left.\mathrm{H}, \mathrm{CH}_{2} \mathrm{CH}_{3}\right) ;{ }^{13} \mathrm{C}$ NMR $\left(62.5 \mathrm{MHz}, \mathrm{CDCl}_{3}\right) \delta 187.6,173.1,171.7,145.3,127.2$, 106.4, 69.9, 65.2, 41.4, 37.8, 36.6, 31.9, 29.6, 29.4, 29.3, 25.5, 22.7, 14.1;

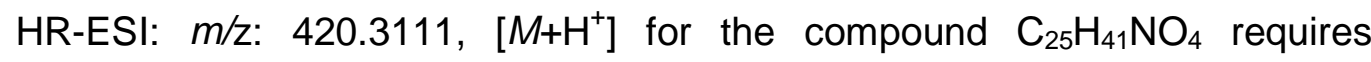
420.3114 . 


\section{Diastereoselective preparation of para-quinol 9b.}

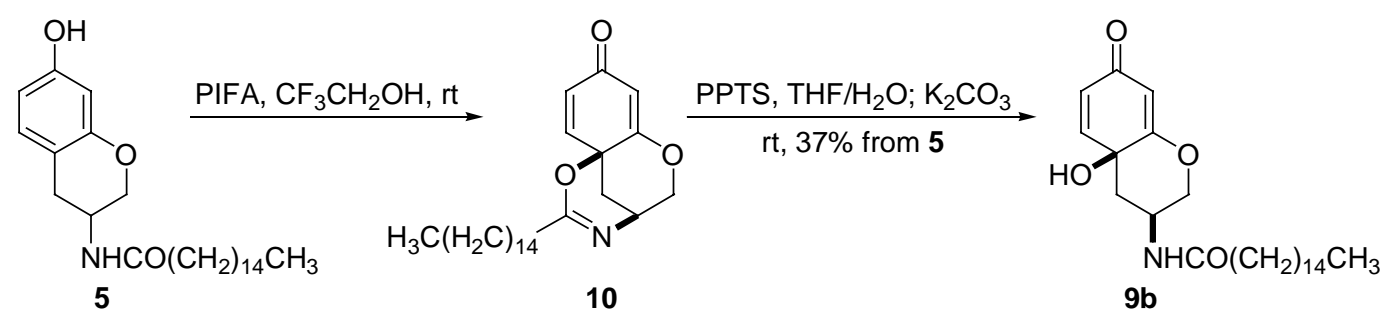

To a vigorously stirred fine suspension of phenol 5 (144.6 $\mathrm{mg}, 0.36 \mathrm{mmol})$ in 2,2,2-trifluoroethanol $(10 \mathrm{~mL}$ ) was added at ambient temperature [bis(trifluoroacetoxy)iodo]benzene (240 $\mathrm{mg}, 0.56 \mathrm{mmol}$ ). The reaction mixture was stirred for $4 \mathrm{~h}$ and then it was concentrated under reduced pressure. The residue thus obtained was dissolved in ethyl acetate $(20 \mathrm{~mL})$, washed sequentially with a saturated aqueous solution of sodium bicarbonate $(10 \mathrm{~mL})$, water $(2 \times$ $10 \mathrm{~mL}$ ) and brine $(10 \mathrm{~mL})$, dried over $\mathrm{Na}_{2} \mathrm{SO}_{4}$ and concentrated under reduced pressure. The residue thus obtained used without further purification in the next step. A small sample of it was subjected to flash column chromatography (3:97 methanol-dichloromethane) to produce compound $\mathbf{1 0}$ as light yellow oil: $R_{\mathrm{f}} 0.21$ (3:97 methanol-dichloromethane); ${ }^{1} \mathrm{H}$ NMR (250 $\left.\mathrm{MHz}, \mathrm{CDCl}_{3}\right) \delta 6.48(\mathrm{~d}, J=9.9 \mathrm{~Hz}, 1 \mathrm{H}, \mathrm{CH}=), 6.24(\mathrm{dd}, J=9.9,1.6 \mathrm{~Hz}, 1 \mathrm{H}$, $\mathrm{CH}=), 5.78(\mathrm{~d}, J=1.6 \mathrm{~Hz}, 1 \mathrm{H}, \mathrm{CH}=), 4.38(\mathrm{brd}, J=10.8 \mathrm{~Hz}, 1 \mathrm{H}, \mathrm{CHHO}$ ), 4.00 (dd, $J=10.8,1.9 \mathrm{~Hz}, 1 \mathrm{H}, \mathrm{CHHO}$ ), 3.88-3.82 (m, $1 \mathrm{H}, \mathrm{CHN}$ ), 2.27 (t, $J=$ $\left.7.6 \mathrm{~Hz}, 2 \mathrm{H}, \mathrm{CH}_{2} \mathrm{C}(\mathrm{O}) \mathrm{N}\right), 2.11-1.98\left(\mathrm{~m}, 2 \mathrm{H}, \mathrm{CH}_{2} \mathrm{CHN}\right), 1.65-1.54(\mathrm{~m}, 2 \mathrm{H}$, $\left.\mathrm{N}(\mathrm{O}) \mathrm{CCH}_{2} \mathrm{CH}_{2}\right), 1.25$ (brs, $\left.24 \mathrm{H},\left(\mathrm{CH}_{2}\right)_{12} \mathrm{CH}_{3}\right), 0.88$ (t, $J=6.4 \mathrm{~Hz}, 3 \mathrm{H}$, $\left.\mathrm{CH}_{2} \mathrm{CH}_{3}\right) ;{ }^{13} \mathrm{C} \mathrm{NMR}\left(62.5 \mathrm{MHz}, \mathrm{CDCl}_{3}\right) \delta 161.8,141.6,130.3,110.0,74.4$, 47.0, 34.7, 31.9, 29.7, 29.5, 29.3, 29.2, 28.9, 26.2, 22.7, 14.1; HR-ESI-TOF: $\mathrm{m} / \mathrm{z}: 402.3008,\left[\mathrm{M}+\mathrm{H}^{+}\right]$for the compound $\mathrm{C}_{25} \mathrm{H}_{39} \mathrm{NO}_{3}$ requires 402.3003 .

The above residue was dissolved in THF $(5 \mathrm{~mL})$ and water $(0.5 \mathrm{~mL})$ and PPTS $(46 \mathrm{mg}$ ) were added to the solution. The mixture was stirred at ambient temperature for $18 \mathrm{~h}$. Solid potassium carbonate $(260 \mathrm{mg}$ ) was added and stirring was continued for $24 \mathrm{~h}$. The reaction mixture was then poured in water $(10 \mathrm{~mL})$ and extracted with ethyl acetate $(3 \times 15 \mathrm{~mL})$. The combined organic phases were washed sequentially with $0.01 \mathrm{~N}$ aqueous $\mathrm{HCl}$ 
solution $(10 \mathrm{~mL})$, water $(10 \mathrm{~mL})$ and brine $(10 \mathrm{~mL})$, dried over $\mathrm{Na}_{2} \mathrm{SO}_{4}$ and concentrated under reduced pressure. The residue thus obtained was purified by flash column chromatography (3:97 methanol-dichloromethane) to produce compound $9 \mathrm{~b}$ as light yellow foam (55.5 mg, 37\%).

\section{Preparation of diol 11.}
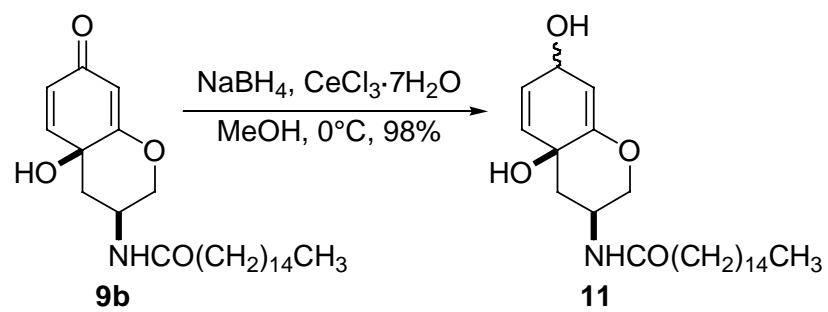

11

To a stirred solution of quinol $9 \mathrm{~b}(166 \mathrm{mg}, 0.40 \mathrm{mmol})$ and $\mathrm{CeCl}_{3} \cdot 7 \mathrm{H}_{2} \mathrm{O}(295$ $\mathrm{mg}, 0.80 \mathrm{mmol})$ in methanol $(10 \mathrm{~mL})$ was added, at $0^{\circ} \mathrm{C}$ and in small portions, sodium borohydride $(23 \mathrm{mg}, 0.6 \mathrm{mmol})$. Upon reaction completion, excess reagent was quenched by addition of acetone $(2 \mathrm{~mL})$. The mixture was stirred at ambient temperature for $30 \mathrm{~min}$ and was then poured in water $(20 \mathrm{~mL})$ and extracted with ethyl acetate $(4 \times 15 \mathrm{~mL})$. The combined organic phases were washed sequentially with water $(2 \times 10 \mathrm{~mL})$ and brine $(2$ $\times 10 \mathrm{~mL}$ ), dried over $\mathrm{Na}_{2} \mathrm{SO}_{4}$ and concentrated under reduced pressure to produce diol 11 (163 mg, 98\%) as a 1:2 mixture of diastereomers. Further purification of this sensitive intermediate is neither necessary nor advisable. Light yellow oil; ${ }^{1} \mathrm{H}$ NMR $\left(250 \mathrm{MHz}, \mathrm{CDCl}_{3}\right) \delta 7.69$, 7.53 (two brd in a ration $1: 2, J=7.5 \mathrm{~Hz}, 1 \mathrm{H}, \mathrm{NHCO}), 6.02-5.86(\mathrm{~m}, 1 \mathrm{H}, \mathrm{CH}=)$, 5.82-5.68 (m, $1 \mathrm{H}$, $\mathrm{CH}=$ ), 5.57-5.44 (m, $1 \mathrm{H}, \mathrm{CH}=), 4.69,4.60$ (two brs in a ratio $2: 1,1 \mathrm{H}$, $\mathrm{CHOH}), 4.34-3.95(\mathrm{~m}, 2 \mathrm{H}, \mathrm{CHNH}+\mathrm{CHHO}), 3.75-3.47(\mathrm{~m}, 1 \mathrm{H}, \mathrm{CH} O)$, 2.29-1.83 (m, $\left.4 \mathrm{H}, \mathrm{CH}_{2} \mathrm{CHNH}+\mathrm{COCH}_{2}\right), 1.73-1.51\left(\mathrm{~m}, 2 \mathrm{H}, \mathrm{COCH}_{2} \mathrm{CH}_{2}\right)$, 1.48-1.11 (m, $\left.24 \mathrm{H},\left(\mathrm{CH}_{2}\right){ }_{12} \mathrm{CH}_{3}\right), 0.87\left(\mathrm{t}, \mathrm{J}=6.5 \mathrm{~Hz}, 3 \mathrm{H}, \mathrm{CH}_{2} \mathrm{CH}_{3}\right) ;{ }^{13} \mathrm{C} \mathrm{NMR}$ $\left(62.5 \mathrm{MHz}, \mathrm{CDCl}_{3}\right) \delta 172.8,132.3,131.6,130.4,111.3,109.6,73.7,64.2$, 43.7, 43.4, 37.4, 37.0, 31.9, 29.6, 29.5, 29.3, 25.6, 22.6, 14.1; HR-ESI-TOF: $\mathrm{m} / \mathrm{z}$ : 422.3258, $\left[\mathrm{M}_{+} \mathrm{H}^{+}\right]$for the compound $\mathrm{C}_{25} \mathrm{H}_{43} \mathrm{NO}_{4}$ requires 422.3265 . 


\section{Preparation of ketal 12a.}

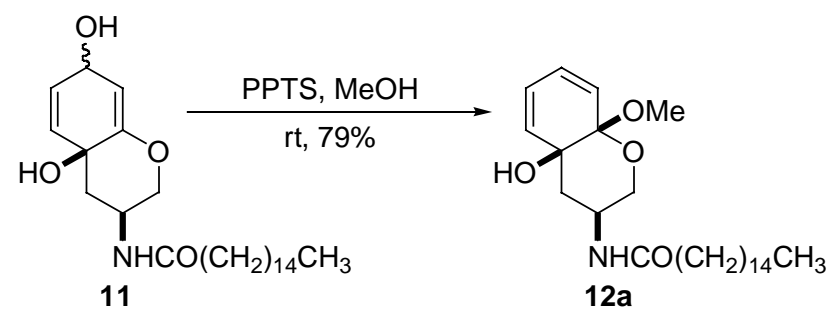

To a stirred solution of diol 11 (55 $\mathrm{mg}, 0.13 \mathrm{mmol})$ in methanol $(15 \mathrm{~mL})$ was added at ambient temperature, PPTS (16 mg, $0.07 \mathrm{mmol})$. The reaction mixture was stirred for $4 \mathrm{~h}$. It was then poured in aqueous solution of sodium bicarbonate $(20 \mathrm{~mL})$ and extracted with ethyl acetate $(4 \times 15 \mathrm{~mL})$. The combined organic phases were washed sequentially with water $(2 \times 10 \mathrm{~mL})$ and brine $(2 \times 10 \mathrm{~mL})$, dried over $\mathrm{Na}_{2} \mathrm{SO}_{4}$ and concentrated under reduced pressure. The residue thus obtained was purified by flash column chromatography (4:6 ethyl acetate-hexane) to produce ketal 12a (44 mg, $79 \%)$ as colorless oil: $R_{\mathrm{f}} 0.25$ (1:1 ethyl acetate-hexane); ${ }^{1} \mathrm{H}$ NMR (500 MHz, $\left.\mathrm{CDCl}_{3}\right) \delta 7.15$ (brd, $J=8.0 \mathrm{~Hz}, 1 \mathrm{H}, \mathrm{NHCO}$ ), 6.18 (ddd, $J=9.9,4.7,1.2 \mathrm{~Hz}, 1$ $\mathrm{H}, \mathrm{CH}=), 5.89$ (d, J = 9.9 Hz, 1 H, CH=), 5.85-5.79 (m, $2 \mathrm{H}, \mathrm{CH}=), 4.07-4.02$ (m, $1 \mathrm{H}, \mathrm{CHNH}$ ), 3.90 (brd, $J=12.1 \mathrm{~Hz}, 1 \mathrm{H}, \mathrm{CHHO}$ ), 3.72 (dd, $J=12.1,1.9$ $\mathrm{Hz}, 1 \mathrm{H}, \mathrm{CH} H \mathrm{O}$ ), 3.37 (brs, $1 \mathrm{H}, \mathrm{OH}$ ), 3.34 (s, $3 \mathrm{H}, \mathrm{OCH}_{3}$ ), 2.16 (t, J = $7.7 \mathrm{~Hz}$, $2 \mathrm{H}, \mathrm{COCH}_{2}$ ), 1.92 (dt, $J=14.7,2.4 \mathrm{~Hz}, 1 \mathrm{H}, \mathrm{CHHCHNH}$ ), 1.83 (dd, $J=14.7$, $4.2 \mathrm{~Hz}, 1 \mathrm{H}, \mathrm{CH} H \mathrm{CHNH}), 1.64-1.56\left(\mathrm{~m}, 2 \mathrm{H}, \mathrm{COCH}_{2} \mathrm{CH}_{2}\right), 1.33-1.19(\mathrm{~m}, 24$ $\left.\mathrm{H},\left(\mathrm{CH}_{2}\right)_{12} \mathrm{CH}_{3}\right), 0.87\left(\mathrm{t}, J=6.7 \mathrm{~Hz}, 3 \mathrm{H}, \mathrm{CH}_{2} \mathrm{CH}_{3}\right) ;{ }^{13} \mathrm{C} \operatorname{NMR}(62.5 \mathrm{MHz}$, $\left.\mathrm{CDCl}_{3}\right) \delta 172.7,139.0,129.7,127.4,121.2,98.2,72.8,66.8,50.1,41.3,36.9$, 35.3, 31.9, 29.6, 29.5, 29.3, 25.6, 22.6, 14.1; HR-ESI-TOF: m/z: 458.3241, $\left[\mathrm{M}+\mathrm{Na}^{+}\right]$for the compound $\mathrm{C}_{26} \mathrm{H}_{45} \mathrm{NO}_{4}$ requires 458.3241 .

\section{Preparation of ketal $\mathbf{1 2 b .}$}

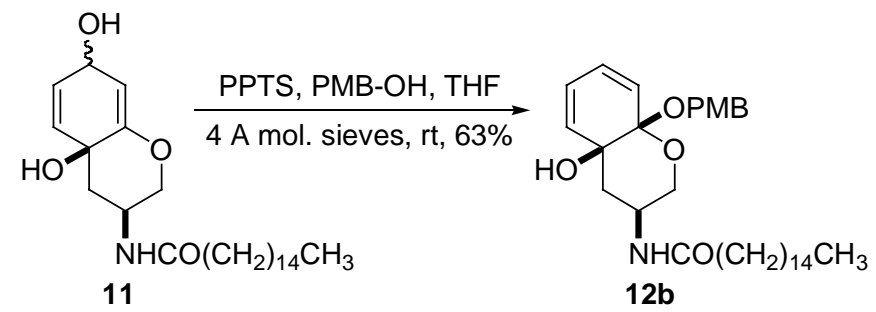

To a stirred solution of diol 11 (163 mg, $0.39 \mathrm{mmol})$ in THF $(7 \mathrm{~mL})$ were added powdered molecular sieves $4 \AA$ (160 mg), 4-methoxybenzyl alcohol 
(280 mg, $2.0 \mathrm{mmol})$ and PPTS (60 mg, $0.2 \mathrm{mmol})$. The mixture was stirred at ambient temperature for $24 \mathrm{~h}$. The reaction was quenched by addition of solid sodium bicarbonate $(100 \mathrm{mg})$. The mixture was diluted with ethyl acetate $(20 \mathrm{~mL})$ and filtered with the aid of Celite ${ }^{\circledR}$. The filtrate was poured in saturated aqueous solution of sodium bicarbonate $(10 \mathrm{~mL})$ and the mixture was extracted with ethyl acetate $(2 \times 15 \mathrm{~mL})$. The combined organic phases were washed sequentially with water $(2 \times 10 \mathrm{~mL})$ and brine $(2 \times 10 \mathrm{~mL})$, dried over $\mathrm{Na}_{2} \mathrm{SO}_{4}$ and concentrated under reduced pressure. The residue thus obtained was purified by flash column chromatography (3:7 ethyl acetatehexane) to produce ketal $\mathbf{1 2 b}(126 \mathrm{mg}, 60 \%)$ as colorless oil: $R_{\mathrm{f}} 0.36(1: 1$ ethyl acetate-hexane); ${ }^{1} \mathrm{H}$ NMR $\left(500 \mathrm{MHz} \mathrm{CDCl}_{3}\right) \delta 7.22(\mathrm{~d}, J=8.4 \mathrm{~Hz}, 2 \mathrm{H}$, ArH), 7.21 (obs $1 \mathrm{H}, \mathrm{NHCO}$ ), 6.86 (d, $J=8.4 \mathrm{~Hz}, 2 \mathrm{H}, \operatorname{ArH}$ ), 6.20 (ddd, $J=$ 9.9, 4.4, 1.4 Hz, $1 \mathrm{H}, \mathrm{CH}=), 6.01$ (d, J = 9.9 Hz, $1 \mathrm{H}, \mathrm{CH}=)$, 5.87-5.82 (m, 2 $\mathrm{H}, \mathrm{CH}=), 4.60\left(\mathrm{AB}_{\mathrm{q}}, J=10.8 \mathrm{~Hz}, \Delta v=82.9 \mathrm{~Hz}, 2 \mathrm{H}, \mathrm{OCH}_{2} \mathrm{Ar}\right), 4.12-4.06(\mathrm{~m}$, $1 \mathrm{H}, \mathrm{CHNH}$ ), 3.95 (brd, J=12.0 Hz, $1 \mathrm{H}, \mathrm{CHHO}$ ), 3.80 (s, $3 \mathrm{H}, \mathrm{OCH}_{3}$ ), 3.79 (obs dd, $J=12.0,1.9 \mathrm{~Hz}, 1 \mathrm{H}, \mathrm{CH} H \mathrm{O}$ ), 3.47 (brs, $1 \mathrm{H}, \mathrm{OH}$ ), 2.19 (t, J = 7.6 $\mathrm{Hz}, 2 \mathrm{H}, \mathrm{COCH}_{2}$ ), 1.95 (brd, $J=14.6 \mathrm{~Hz}, 1 \mathrm{H}, \mathrm{CHHCHNH}$ ), 1.87 (dd, J = 14.6, $4.5 \mathrm{~Hz}, 1 \mathrm{H}, \mathrm{CH} H \mathrm{CHNH}), 1.66-1.61\left(\mathrm{~m}, 2 \mathrm{H}, \mathrm{COCH}_{2} \mathrm{CH}_{2}\right), 1.36-1.22$ (m, 24 $\left.\mathrm{H},\left(\mathrm{CH}_{2}\right)_{12} \mathrm{CH}_{3}\right), 0.89\left(\mathrm{t}, J=6.8 \mathrm{~Hz}, 3 \mathrm{H}, \mathrm{CH}_{2} \mathrm{CH}_{3}\right) ;{ }^{13} \mathrm{C} \mathrm{NMR}(62.5 \mathrm{MHz}$, $\left.\mathrm{CDCl}_{3}\right) \delta 172.6,159.1,139.1,130.2,129.5,129.3,127.7,121.2,113.6,98.2$, 72.7, 66.7, 64.1, 55.1, 41.3, 36.9, 35.2, 31.6, 29.6, 29.5, 29.3, 29.2, 25.6, 22.6, 14.1; HR-ESI-TOF: $m / z$ : 542.3829, $\left[M+\mathrm{H}^{+}\right]$for the compound $\mathrm{C}_{33} \mathrm{H}_{51} \mathrm{NO}_{5}$ requires 542.3840 .

\section{Preparation of epoxides 13a and 14a.}
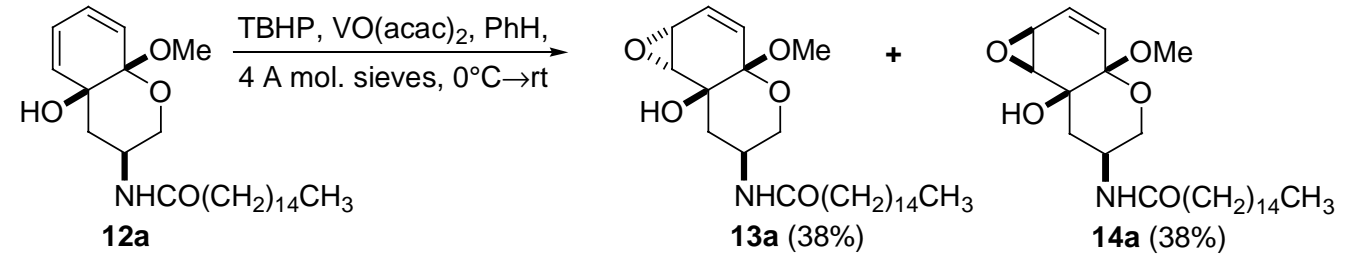

To a stirred solution of ketal $12 \mathbf{a}(20 \mathrm{mg}, 46 \mu \mathrm{mol})$ in benzene $(3 \mathrm{~mL})$ were added $0.02 \mathrm{M}$ solution of $\mathrm{VO}(\mathrm{acac})_{2}$ in benzene $(0.3 \mathrm{~mL}, 6 \mu \mathrm{mol})$ and powdered molecular sieves $4 \AA$ (30 mg). The mixture was cooled at $0{ }^{\circ} \mathrm{C}$ and then $5.5 \mathrm{M}$ solution of TBHP in decane $(20 \mu \mathrm{L}, 0.1 \mathrm{mmol})$ was added. The 
reaction mixture was stirred at $0^{\circ} \mathrm{C}$ for one hour and then at ambient temperature for $24 \mathrm{~h}$. Upon reaction completion, the molecular sieves were removed by filtration with the aid of Celite $\AA$ and the filtrate was concentrated under reduced pressure. The residue thus obtained was purified by flash column chromatography (1:9 $\rightarrow$ 1:1 ethyl acetate-hexane) to produce, in order of elution:

compound 13a as white wax: $7.1 \mathrm{mg}(34 \%): R_{\mathrm{f}} 0.13$ (1:1 ethyl acetatehexane); ${ }^{1} \mathrm{H}$ NMR $\left(500 \mathrm{MHz}, \mathrm{CDCl}_{3}\right) \delta 6.13(\mathrm{dd}, J=9.7,4.0 \mathrm{~Hz}, 1 \mathrm{H}$, $\mathrm{CHCH}=\mathrm{CH}$ ), 6.00 (dd, $J=9.7,1.7 \mathrm{~Hz}, 1 \mathrm{H}, \mathrm{CHCH}=\mathrm{CH}), 5.22(\mathrm{brd}, J=7.7 \mathrm{~Hz}$, $1 \mathrm{H}, \mathrm{NHCO}), 4.05-3.92(\mathrm{~m}, 1 \mathrm{H}, \mathrm{CHNH}), 3.79-3.75(\mathrm{~m}, 2 \mathrm{H}, \mathrm{CH} \mathrm{HO}+$ $\mathrm{CH}(\mathrm{O}) \mathrm{CHCH}=\mathrm{CH}), 3.57\left(\mathrm{~s}, 3 \mathrm{H}, \mathrm{OCH}_{3}\right), 3.47(\mathrm{~d}, J=7.3 \mathrm{~Hz}, 1 \mathrm{H}, \mathrm{CHCH}=\mathrm{CH})$, $3.18(\mathrm{t}, J=10.6 \mathrm{~Hz}, 1 \mathrm{H}, \mathrm{CHHO}$ ), 2.81 (brs, $1 \mathrm{H}, \mathrm{OH}), 2.14(\mathrm{t}, J=7.6 \mathrm{~Hz}, 2 \mathrm{H}$, $\left.\mathrm{COCH}_{2}\right), 1.92-1.84\left(\mathrm{~m}, 2 \mathrm{H}, \mathrm{CH}_{2} \mathrm{CHNH}\right), 1.63-1.58\left(\mathrm{~m}, 2 \mathrm{H}, \mathrm{COCH}_{2} \mathrm{CH}_{2}\right)$, 1.32-1.20 (m, $\left.24 \mathrm{H},\left(\mathrm{CH}_{2}\right){ }_{12} \mathrm{CH}_{3}\right), 0.88\left(\mathrm{t}, \mathrm{J}=6.9 \mathrm{~Hz}, 3 \mathrm{H}, \mathrm{CH}_{2} \mathrm{CH}_{3}\right)$; HR-ESITOF: $\mathrm{m} / \mathrm{z}: 474.3184,\left[\mathrm{M}+\mathrm{Na}^{+}\right]$for the compound $\mathrm{C}_{26} \mathrm{H}_{45} \mathrm{NO}_{5}$ requires 474.3190. and

compound 14a as white wax: $7.1 \mathrm{mg}(34 \%)$ : $R_{\mathrm{f}} 0.07$ (1:1 ethyl acetatehexane); ${ }^{1} \mathrm{H}$ NMR (500 MHz, $\left.\mathrm{CDCl}_{3}\right) \delta 7.05$ (brd, $J=8.5 \mathrm{~Hz}, 1 \mathrm{H}, \mathrm{NHCO}$ ), $6.37(\mathrm{dd}, J=10.1,3.8 \mathrm{~Hz}, 1 \mathrm{H}, \mathrm{CHCH}=\mathrm{CH}), 5.94(\mathrm{~d}, J=10.1 \mathrm{~Hz}, 1 \mathrm{H}$, $\mathrm{CHCH}=\mathrm{CH}), 4.17-4.12(\mathrm{~m}, 1 \mathrm{H}, \mathrm{CHNH}), 3.84(\mathrm{dt}, J=12.1,2.5 \mathrm{~Hz}, 1 \mathrm{H}$, $\mathrm{CHHO}$ ), $3.74(\mathrm{~d}, J=2.4 \mathrm{~Hz}, 1 \mathrm{H}, \mathrm{OH}$ ), $3.55(\mathrm{dd}, J=12.1,2.7 \mathrm{~Hz}, 1 \mathrm{H}$, $\mathrm{CHHO}$ ), 3.41-3.38 (m, $1 \mathrm{H}, \mathrm{CHCH}=\mathrm{CH}), 3.38\left(\mathrm{~s}, 3 \mathrm{H}, \mathrm{OCH}_{3}\right), 3.28(\mathrm{~d}, J=4.0$ $\mathrm{Hz}, 1 \mathrm{H}, \mathrm{CH}(\mathrm{O}) \mathrm{CHCH}=\mathrm{CH}$ ), $2.18\left(\mathrm{t}, J=7.7 \mathrm{~Hz}, 2 \mathrm{H}, \mathrm{COCH}_{2}\right), 1.96$ (dt, $J=$ 14.6, $2.3 \mathrm{~Hz}, 1 \mathrm{H}, \mathrm{CHHCHNH}), 1.66-1.59\left(\mathrm{~m}, 3 \mathrm{H}, \mathrm{CH} H \mathrm{CHNH}+\mathrm{COCH}_{2} \mathrm{CH}_{2}\right.$ ), 1.32-1.24 (m, $\left.24 \mathrm{H},\left(\mathrm{CH}_{2}\right){ }_{12} \mathrm{CH}_{3}\right), 0.88\left(\mathrm{t}, \mathrm{J}=6.8 \mathrm{~Hz}, 3 \mathrm{H}, \mathrm{CH}_{2} \mathrm{CH}_{3}\right) ;{ }^{13} \mathrm{C} \mathrm{NMR}$ $\left(62.5 \mathrm{MHz}, \mathrm{CDCl}_{3}\right) \delta 172.6,133.2,131.2,96.2,70.8,66.5,58.3,50.9,48.2$, 41.4, 36.9, 33.2, 31.9, 29.6, 29.5, 29.3, 25.6, 22.7, 14.1; HR-ESI-TOF: m/z: 474.3186, $\left[\mathrm{M}+\mathrm{Na}^{+}\right]$for the compound $\mathrm{C}_{26} \mathrm{H}_{45} \mathrm{NO}_{5}$ requires 474.3190 . 
Preparation of epoxides $13 \mathrm{~b}$ and $14 \mathrm{~b}$.
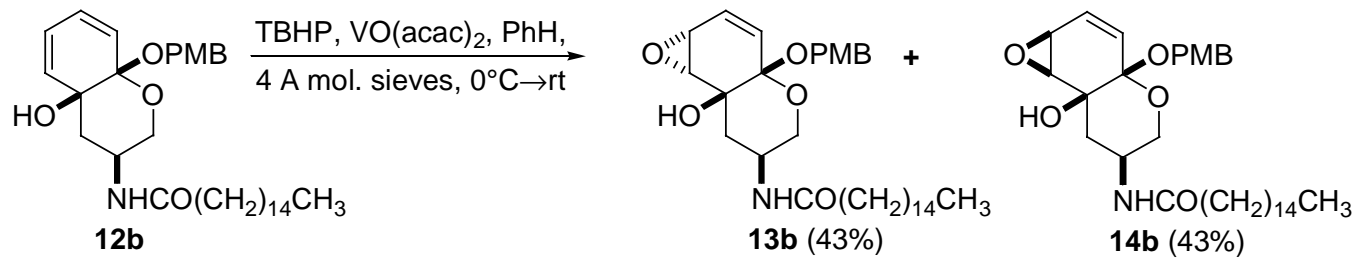

Oxidation of ketal 12b (120 mg, $0.22 \mathrm{mmol}$ ), using the protocol described above, produces:

Compound 13b as amorphous white solid: $52.8 \mathrm{mg}(43 \%) ; R_{\mathrm{f}} 0.26$ (1:1 ethyl acetate-hexane); ${ }^{1} \mathrm{H}$ NMR $\left(500 \mathrm{MHz}, \mathrm{CDCl}_{3}\right) \delta 7.36(\mathrm{~d}, J=7.9 \mathrm{~Hz}, 2 \mathrm{H}, \mathrm{ArH})$, $6.90(\mathrm{~d}, J=7.9 \mathrm{~Hz}, 2 \mathrm{H}, \mathrm{ArH}), 6.12$ (dd, $J=9.6,2.7 \mathrm{~Hz}, 1 \mathrm{H}, \mathrm{CHCH}=\mathrm{CH}$ ), 6.00 (d, J = 9.6 Hz, $1 \mathrm{H}, \mathrm{CHCH}=\mathrm{CH}$ ), 5.29 (brd, J = $7.7 \mathrm{~Hz}, 1 \mathrm{H}, \mathrm{NHCO}$ ), 4.82 $\left(\mathrm{AB}_{\mathrm{q}}, J=11.5 \mathrm{~Hz}, \Delta \mathrm{v}=67.8 \mathrm{~Hz}, 2 \mathrm{H}, \mathrm{OCH}_{2} \mathrm{Ar}\right), 4.03-3.94(\mathrm{~m}, 1 \mathrm{H}, \mathrm{CHNH})$, $3.81\left(\mathrm{~s}, 3 \mathrm{H}, \mathrm{OCH}_{3}\right), 3.81-3.74(\mathrm{~m}, 2 \mathrm{H}, \mathrm{CH} \mathrm{HO}+\mathrm{CH}(\mathrm{O}) \mathrm{CHCH}=\mathrm{CH}), 3.50$ (brs, $1 \mathrm{H}, \mathrm{CHCH}=\mathrm{CH}$ ), 3.24 (dd, $J=10.7,10.5 \mathrm{~Hz}, 1 \mathrm{H}, \mathrm{CH} \mathrm{HO}$ ), 2.88 (brs, 1 $\mathrm{H}, \mathrm{OH}$ ), 2.11 (t, $J=7.3 \mathrm{~Hz}, 2 \mathrm{H}, \mathrm{COCH}_{2}$ ), 1.92 (dd, $J=12.2,11.9 \mathrm{~Hz}, 1 \mathrm{H}$, $\mathrm{CHHCHNH}$ ), 1.85 (brd, $J=11.9$ hz, $1 \mathrm{H}, \mathrm{CHHCHNH}), 1.62-1.54$ (m, $2 \mathrm{H}$, $\left.\mathrm{COCH}_{2} \mathrm{CH}_{2}\right), 1.33-1.19\left(\mathrm{~m}, 24 \mathrm{H},\left(\mathrm{CH}_{2}\right)_{12} \mathrm{CH}_{3}\right), 0.87(\mathrm{t}, J=6.5 \mathrm{~Hz}, 3 \mathrm{H}$, $\left.\mathrm{CH}_{2} \mathrm{CH}_{3}\right) ;{ }^{13} \mathrm{C}$ NMR $\left(62.5 \mathrm{MHz}, \mathrm{CDCl}_{3}\right) \delta 172.7,159.3,140.2,130.1,129.5$, 128.7, 125.4, 113.7, 96.3, 69.6, 63.5, 62.4, 55.3, 54.4, 50.2, 43.5, 37.6, 36.7, 31.9, 29.7, 29.5, 29.3, 29.2, 25.6, 22.7, 14.1; HR-ESI-TOF: m/z: 558.3785, $\left[\mathrm{M}+\mathrm{H}^{+}\right]$for the compound $\mathrm{C}_{33} \mathrm{H}_{51} \mathrm{NO}_{6}$ requires 558.3789, and

Compound 14b as amorphous white solid: $52.8 \mathrm{mg}(43 \%) ; R_{\mathrm{f}} 0.11$ (1:1 ethyl acetate-hexane); ${ }^{1} \mathrm{H}$ NMR $\left(500 \mathrm{MHz}, \mathrm{CDCl}_{3}\right) \delta 7.29(\mathrm{~d}, J=8.4 \mathrm{~Hz}, 2 \mathrm{H}, \mathrm{ArH})$, 7.06 (brd, $J=8.5 \mathrm{~Hz}, 1 \mathrm{H}, \mathrm{NHCO}), 6.86$ (d, J = 8.4 Hz, $2 \mathrm{H}, \mathrm{ArH}), 6.34$ (dd, J $=10.1,3.8 \mathrm{~Hz}, 1 \mathrm{H}, \mathrm{CHCH}=\mathrm{CH}), 5.99(\mathrm{~d}, \mathrm{~J}=10.1 \mathrm{~Hz}, 1 \mathrm{H}, \mathrm{CHCH}=\mathrm{CH}), 4.58$ $\left(\mathrm{AB}_{\mathrm{q}}, J=10.4 \mathrm{~Hz}, \Delta \mathrm{v}=63.2 \mathrm{~Hz}, 2 \mathrm{H}, \mathrm{OCH}_{2} \mathrm{Ar}\right), 4.19-4.14(\mathrm{~m}, 1 \mathrm{H}, \mathrm{CHNH})$, 3.85 (dt, $J=12.1,2.0 \mathrm{~Hz}, 1 \mathrm{H}, \mathrm{CH} \mathrm{HO}$ ), 3.80 (d, J = 2.5 Hz, $1 \mathrm{H}, \mathrm{OH}$ ), 3.79 (s, $\left.3 \mathrm{H}, \mathrm{OCH}_{3}\right), 3.58(\mathrm{dd}, \mathrm{J}=12.1,2.7 \mathrm{~Hz}, 1 \mathrm{H}, \mathrm{CH} \mathrm{O}), 3.41-3.38(\mathrm{~m}, 1 \mathrm{H}$, $\mathrm{CHCH}=\mathrm{CH}$ ), $3.28(\mathrm{~d}, J=4.0 \mathrm{~Hz}, 1 \mathrm{H}, \mathrm{CH}(\mathrm{O}) \mathrm{CHCH}=\mathrm{CH}), 2.17$ (t, J = $7.6 \mathrm{~Hz}, 2$ $\mathrm{H}, \mathrm{COCH}_{2}$ ), 1.95 (brd, $\left.J=14.6 \mathrm{~Hz}, 1 \mathrm{H}, \mathrm{CHHCHNH}\right), 1.68-1.57(\mathrm{~m}, 3 \mathrm{H}$, $\left.\mathrm{CHHCHNH}+\mathrm{COCH}_{2} \mathrm{CH}_{2}\right), 1.34-1.21\left(\mathrm{~m}, 24 \mathrm{H},\left(\mathrm{CH}_{2}\right)_{12} \mathrm{CH}_{3}\right), 0.87(\mathrm{t}, J=6.8$ $\left.\mathrm{Hz}, 3 \mathrm{H}, \mathrm{CH}_{2} \mathrm{CH}_{3}\right) ;{ }^{13} \mathrm{C}$ NMR $\left(62.5 \mathrm{MHz}, \mathrm{CDCl}_{3}\right) \delta 172.5,159.2,133.6,130.9$, 130.0, 129.5, 113.6, 96.3, 70.8, 66.3, 65.1, 58.3, 55.2, 48.2, 41.5, 36.9, 33.1, 
31.7, 29.7, 29.5, 29.3, 25.6, 22.7, 14.1; HR-ESI-TOF: $\mathrm{m} / \mathrm{z}:$ 558.3780, $\left[\mathrm{M}^{+} \mathrm{H}^{+}\right]$ for the compound $\mathrm{C}_{33} \mathrm{H}_{51} \mathrm{NO}_{6}$ requires 558.3789 .

\section{Preparation of acetal 15.}
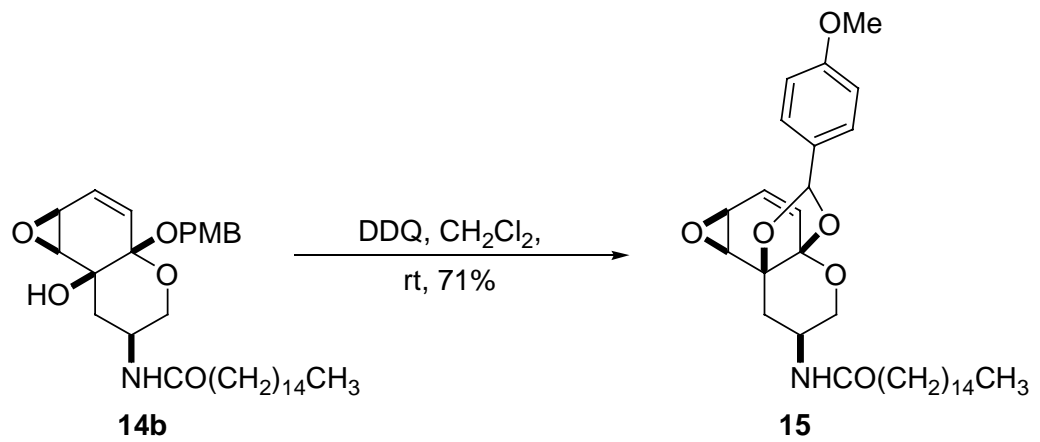

To a stirred solution of compound $14 \mathrm{~b}$ ( $35.5 \mathrm{mg}, 64 \mu \mathrm{mol})$ in dichloromethane $(5 \mathrm{~mL})$ was added, under an atmosphere of argon, DDQ (20 mg, $88 \mu \mathrm{mol})$. The mixture was stirred at ambient temperature for $24 \mathrm{~h}$ and it was then poured in aqueous saturated solution of sodium bicarbonate $(10 \mathrm{~mL})$. The mixture was extracted with ethyl acetate $(2 \times 15 \mathrm{~mL})$. The combined organic phases were washed sequentially with water $(2 \times 10 \mathrm{~mL})$ and brine $(2$ $\times 10 \mathrm{~mL}$ ) and were then dried over $\mathrm{Na}_{2} \mathrm{SO}_{4}$. Evaporation under reduced pressure and further chromatographic purification (1:1 ethyl acetate-hexane) of the residue produced compound $\mathbf{1 5}$ as amorphous white solid $(25.2 \mathrm{mg}$, $71 \%): R_{\mathrm{f}} 0.57$ (4:1 ethyl acetate-acetone); ${ }^{1} \mathrm{H}$ NMR $\left(500 \mathrm{MHz}, \mathrm{CDCl}_{3}\right) \delta 7.59$ (d, $J=8.6 \mathrm{~Hz}, 2 \mathrm{H}, \operatorname{ArH}), 6.92(\mathrm{~d}, J=8.6 \mathrm{~Hz}, 2 \mathrm{H}, \operatorname{ArH}), 6.60$ (brd, $J=7.1 \mathrm{~Hz}$, $1 \mathrm{H}, \mathrm{CHNHCO}$ ), 6.54 (dd, $J=10.0,3.8 \mathrm{~Hz}, 1 \mathrm{H}, \mathrm{CHCH}=\mathrm{CH}$ ), $6.27(\mathrm{~s}, 1 \mathrm{H}$, OCHO), 6.04 (dd, $J=10.0,1.1 \mathrm{~Hz}, \mathrm{CHCH}=\mathrm{CH}), 4.10-4.05(\mathrm{~m}, 1 \mathrm{H}, \mathrm{CHNH})$, 4.04 (dd, $J=15.2,1.1 \mathrm{~Hz}, 1 \mathrm{H}, \mathrm{CHHO}$ ), $3.81\left(\mathrm{~s}, 3 \mathrm{H}, \mathrm{OCH}_{3}\right.$ ), 3.57-3.53 (m, 1 $\mathrm{H}, \mathrm{CHCH}=\mathrm{CH}$ ), $3.47(\mathrm{~d}, J=15.2 \mathrm{~Hz}, 1 \mathrm{H}, \mathrm{CH} H \mathrm{O}), 3.46(\mathrm{~s}, 1 \mathrm{H}, \mathrm{CH}(\mathrm{O}) \mathrm{CH})$, 2.37 (brd, $J=15.1 \mathrm{~Hz}, 1 \mathrm{H}, \mathrm{CHHCHNH}$ ), 2.23 (t, $J=7.6 \mathrm{~Hz}, 2 \mathrm{H}, \mathrm{NHCOCH}_{2}$ ), 1.75 (dd, $J=15.1,4.9 \mathrm{~Hz}, \mathrm{CH} H C H N H), 1.68-1.62\left(\mathrm{~m}, 2 \mathrm{H}, \mathrm{NHCOCH}_{2} \mathrm{CH}_{2}\right.$ ), 1.38-1.21 (m, $\left.24 \mathrm{H},\left(\mathrm{CH}_{2}\right){ }_{12} \mathrm{CH}_{3}\right), 0.88\left(\mathrm{t}, \mathrm{J}=6.8 \mathrm{~Hz}, 3 \mathrm{H}, \mathrm{CH}_{2} \mathrm{CH}_{3}\right) ;{ }^{13} \mathrm{C} \mathrm{NMR}$ $\left(62.5 \mathrm{MHz}, \mathrm{CDCl}_{3}\right) \delta 172.7,160.8,132.8,131.4,129.5,129.0,113.9,101.8$, 99.4, 66.0, 57.6, 55.3, 49.1, 41.8, 36.9, 31.9, 31.7, 29.7, 29.4, 25.7, 22.7, 14.1; HR-ESI-TOF: $\mathrm{m} / \mathrm{z}: 578.3448,\left[\mathrm{M}+\mathrm{Na}^{+}\right]$for compound $\mathrm{C}_{33} \mathrm{H}_{49} \mathrm{NO}_{6}$ requires 578.3452 . 


\section{Preparation of palmitoyl analogue of scyphostatin 16.}

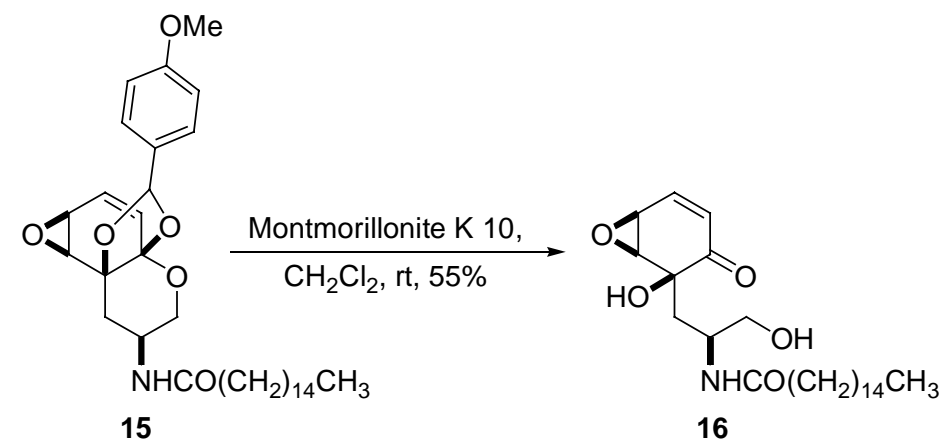

To a solution of $23 \mathrm{mg}(41 \mu \mathrm{mol})$ of compound 15 in dichloromethane $(3 \mathrm{~mL})$ was added montmorillonite K $10(150 \mathrm{mg})$. The mixture was stirred at ambient temperature for $1 \mathrm{~h}$ and was then filtered. The clay was washed on the filter with acetone $(3 \times 10 \mathrm{~mL})$ and the combined filtrates were evaporated under reduced pressure. The residue thus obtained was purified by flash column chromatography (4:1 ethyl acetate-acetone) to produce, in order of elution, unreacted starting material $(8.8 \mathrm{mg})$ and the palmitoyl analogue of scyphostatin $16(6.1 \mathrm{mg}, 55 \%)$ as amorphous white solid: $R_{\mathrm{f}} 0.32$ (4:1 ethyl acetate-acetone); ${ }^{1} \mathrm{H}$ NMR (500 MHz, $\left.\mathrm{CD}_{3} \mathrm{OD}\right) \delta 7.17$ (dd, $J=9.9,3.9 \mathrm{~Hz}, 1$ $\mathrm{H}, \mathrm{CHCH}=\mathrm{CH}$ ), $6.10(\mathrm{dd}, J=9.9,1.6 \mathrm{~Hz}, 1 \mathrm{H}, \mathrm{CHCH}=\mathrm{CH}), 3.99-4.04(\mathrm{~m}, 1$ $\mathrm{H}, \mathrm{CHNH}$ ), $3.66(\mathrm{~d}, J=4.0 \mathrm{~Hz}, 1 \mathrm{H}, \mathrm{CH}(\mathrm{O}) \mathrm{CHCH}=\mathrm{CH}$ ), 3.61 (dt, $J=4.0,1.6$ $\mathrm{Hz}, 1 \mathrm{H}, \mathrm{CH}(\mathrm{O}) \mathrm{CHCH}=\mathrm{CH}$ ), $3.48(\mathrm{dd}, J=10.9,5.2 \mathrm{~Hz}, 1 \mathrm{H}, \mathrm{CHHOH}), 3.41$ (dd, $J=10.9,6.0 \mathrm{~Hz}, 1 \mathrm{H}, \mathrm{CHHOH}$ ), $2.11(\mathrm{dt}, J=7.6,2.1 \mathrm{~Hz}, 2 \mathrm{H}$, $\mathrm{COCH}_{2} \mathrm{CH}_{2}$ ), 2.05 (dd, $J=14.7,3.4 \mathrm{~Hz}, 1 \mathrm{H}, \mathrm{CHHCHNH}$ ), 1.81 (dd, $J=14.7$, $9.4 \mathrm{~Hz}, 1 \mathrm{H}, \mathrm{CH} H \mathrm{CHNH}), 1.55-1.61\left(\mathrm{~m}, 2 \mathrm{H}, \mathrm{COCH}_{2} \mathrm{CH}_{2}\right), 1.25-1.31(\mathrm{~m}, 24$ $\left.\mathrm{H}, \mathrm{CH}_{2}\left(\mathrm{CH}_{2}\right)_{12} \mathrm{CH}_{3}\right), 0.90$ (t, $\left.\mathrm{J}=6.9 \mathrm{~Hz}, 3 \mathrm{H}, \mathrm{CH}_{2} \mathrm{CH}_{3}\right) ;{ }^{13} \mathrm{C} \mathrm{NMR}(62.5 \mathrm{MHz}$, $\left.\mathrm{CD}_{3} \mathrm{OD}\right) \delta 199.5,175.9,145.8,132.0,77.5,65.5,58.2,47.7,39.5,37.1,33.1$, 30.8, 30.7, 30.5, 30.4, 26.7, 23.8, 14.5; HR-ESI-TOF: m/z: 438.3219, $\left[{\left.\mathrm{M}+\mathrm{H}^{+}\right]}^{+}\right.$ for the compound $\mathrm{C}_{25} \mathrm{H}_{43} \mathrm{NO}_{5}$ requires 438.3214 .

\section{Preparation of acetate derivative 17.}

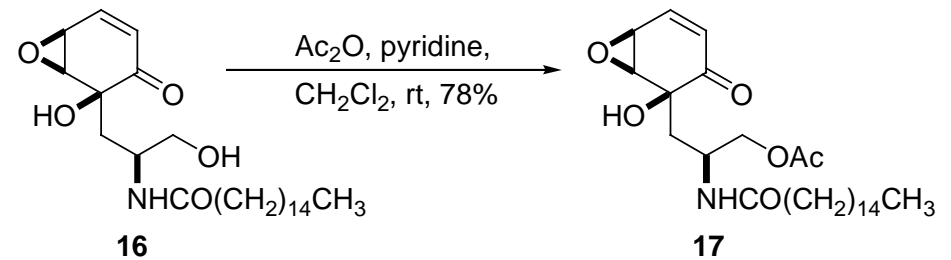


A mixture of compound $16(2.4 \mathrm{mg}, 5.5 \mu \mathrm{mol})$, acetic anhydride ( $50 \mu \mathrm{L}, 0.53$ mmol) and pyridine $(50 \mu \mathrm{L}, 0.63 \mathrm{mmol})$ in dichloromethane $(0.5 \mathrm{~mL})$ was stirred at ambient temperature and under an atmosphere of argon for $12 \mathrm{~h}$. Upon reaction completion, a saturated aqueous solution of sodium bicarbonate $(0.5 \mathrm{~mL})$ was added to the mixture and stirring was continued for $1 \mathrm{~h}$. The mixture was diluted with ethyl acetate $(5 \mathrm{~mL})$ and washed sequentially with water $(5 \mathrm{ml})$, saturated aqueous solution of $\mathrm{CuSO}_{4}(5 \mathrm{~mL})$ and brine $(5 \mathrm{~mL})$. The organic phase was dried over $\mathrm{Na}_{2} \mathrm{SO}_{4}$ and evaporated under reduced pressure. The residue thus obtained was purified by flash column chromatography (6:4 ethyl acetate-hexane) to produce acetate derivative 17 (2.0 mg, 78\%) as white wax: $R_{\mathrm{f}} 0.07$ (1:1 ethyl acetate-hexane); ${ }^{1} \mathrm{H}$ NMR $\left(500 \mathrm{MHz}, \mathrm{CDCl}_{3}\right) \delta 7.15(\mathrm{dd}, J=9.9,3.9 \mathrm{~Hz}, 1 \mathrm{H}, \mathrm{CHCH}=\mathrm{CH}), 6.23$ (dd, $J=9.9,1.5 \mathrm{~Hz}, 1 \mathrm{H}, \mathrm{CHCH}=\mathrm{CH}$ ), 5.83 (brd, $J=7.0 \mathrm{~Hz}, \mathrm{NHCO}$ ), 4.19$4.27(\mathrm{~m}, 2 \mathrm{H}, \mathrm{CHHOAc}+\mathrm{CHNH}), 4.10(\mathrm{dd}, J=10.7,3.9 \mathrm{~Hz}, 1 \mathrm{H}, \mathrm{CH} H \mathrm{OAc})$, $3.98(\mathrm{~s}, 1 \mathrm{H}, \mathrm{COH}), 3.72(\mathrm{~d}, J=3.8 \mathrm{~Hz}, 1 \mathrm{H}, \mathrm{CH}(\mathrm{O}) \mathrm{CHCH}=\mathrm{CH}), 3.58(\mathrm{dt}, J=$ 3.9, $1.6 \mathrm{~Hz}, 1 \mathrm{H}, \mathrm{CH}(\mathrm{O}) \mathrm{CHCH}=\mathrm{CH}), 2.08-2.18\left(\mathrm{~m}, 2 \mathrm{H}, \mathrm{COCH}_{2} \mathrm{CH}_{2}\right), 2.07$ (s, $3 \mathrm{H}, \mathrm{OCOCH}_{3}$ ), 1.94 (dd, $\left.J=14.8,4.6 \mathrm{~Hz}, 1 \mathrm{H}, \mathrm{CHHCHNH}\right), 1.87$ (dd, $J=$ 14.7, $8.3 \mathrm{~Hz}, 1 \mathrm{H}, \mathrm{CH} H \mathrm{CHNH}), 1.56-1.64\left(\mathrm{~m}, 2 \mathrm{H}, \mathrm{COCH}_{2} \mathrm{CH}_{2}\right), 1.22-1.30$ $\left(\mathrm{m}, 24 \mathrm{H}, \mathrm{CH}_{2}\left(\mathrm{CH}_{2}\right){ }_{12} \mathrm{CH}_{3}\right), 0.88\left(\mathrm{t}, J=6.9 \mathrm{~Hz}, 3 \mathrm{H}, \mathrm{CH}_{2} \mathrm{CH}_{3}\right) ;{ }^{13} \mathrm{C} \mathrm{NMR}(62.5$ $\left.\mathrm{MHz}, \mathrm{CDCl}_{3}\right) \delta 197.4,172.9,171.0,144.6,130.0,76.5,65.8,55.7,47.9,44.9$, 37.4, 36.5, 31.4, 29.5, 25.4, 22.6, 20.9, 14.2; HR-ESI-TOF: $\mathrm{m} / \mathrm{z}:$ 480.3326, $\left[\mathrm{M}^{+} \mathrm{H}^{+}\right]$for the compound $\mathrm{C}_{27} \mathrm{H}_{45} \mathrm{NO}_{6}$ requires 480.3319 . 


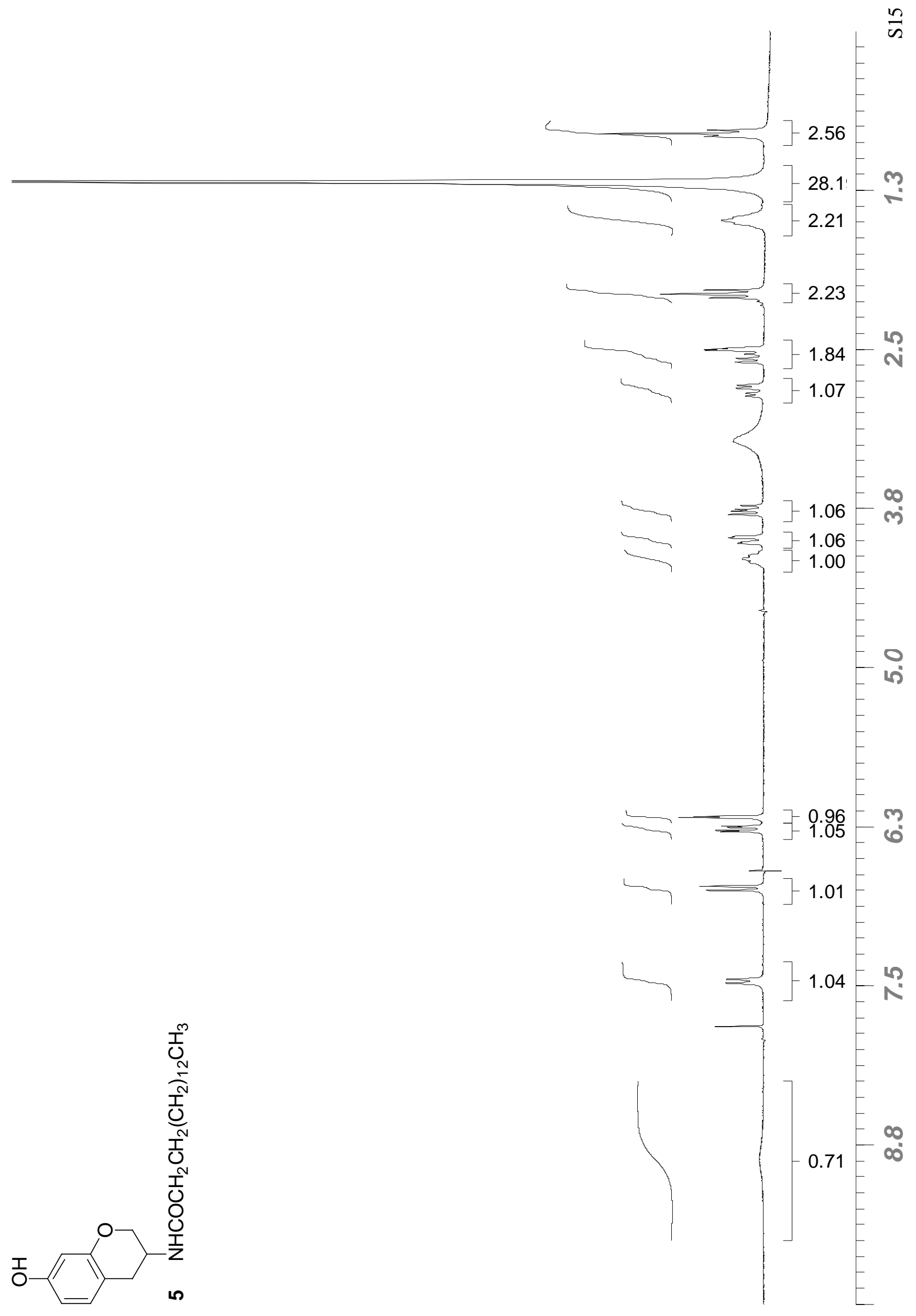




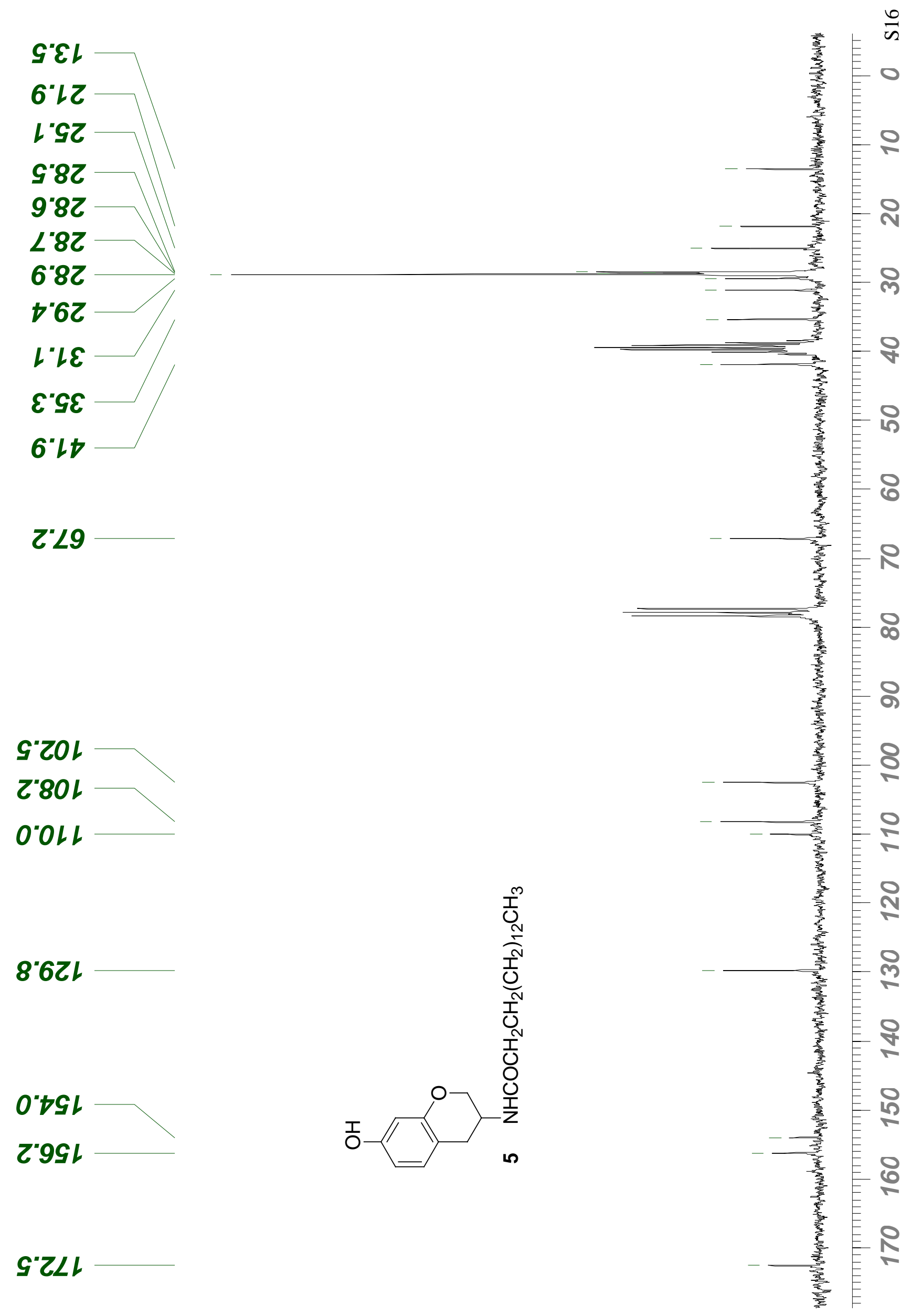




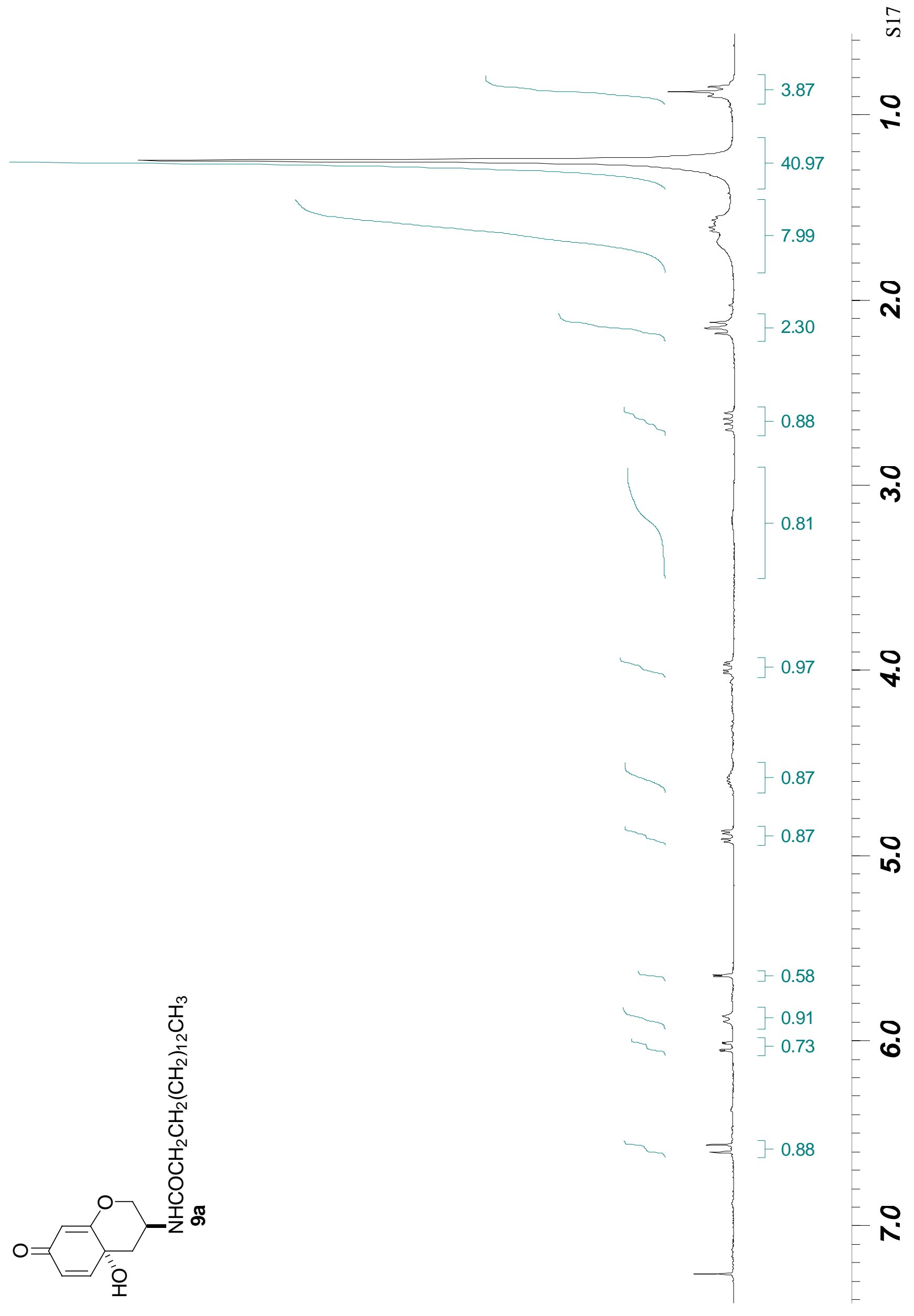




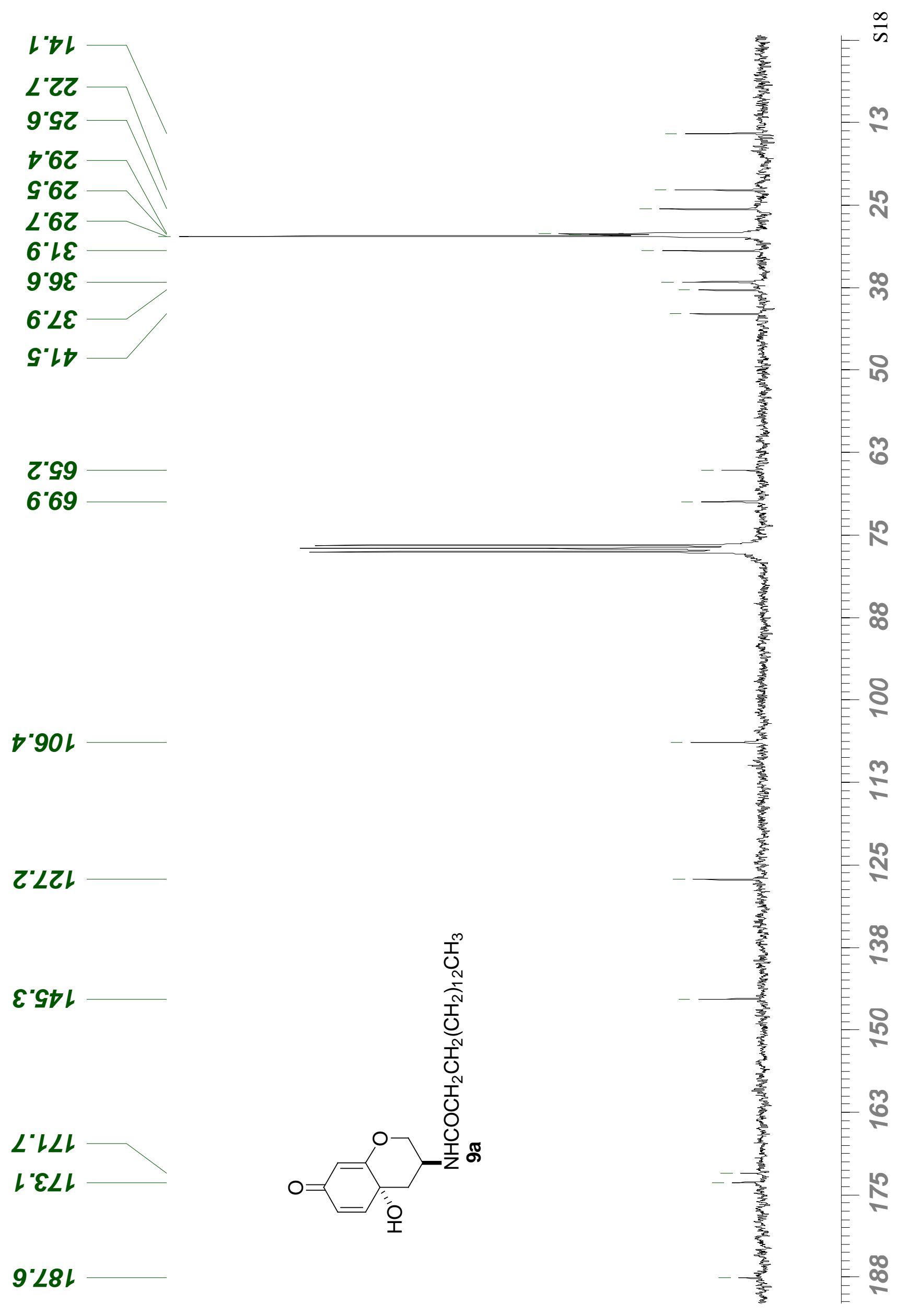




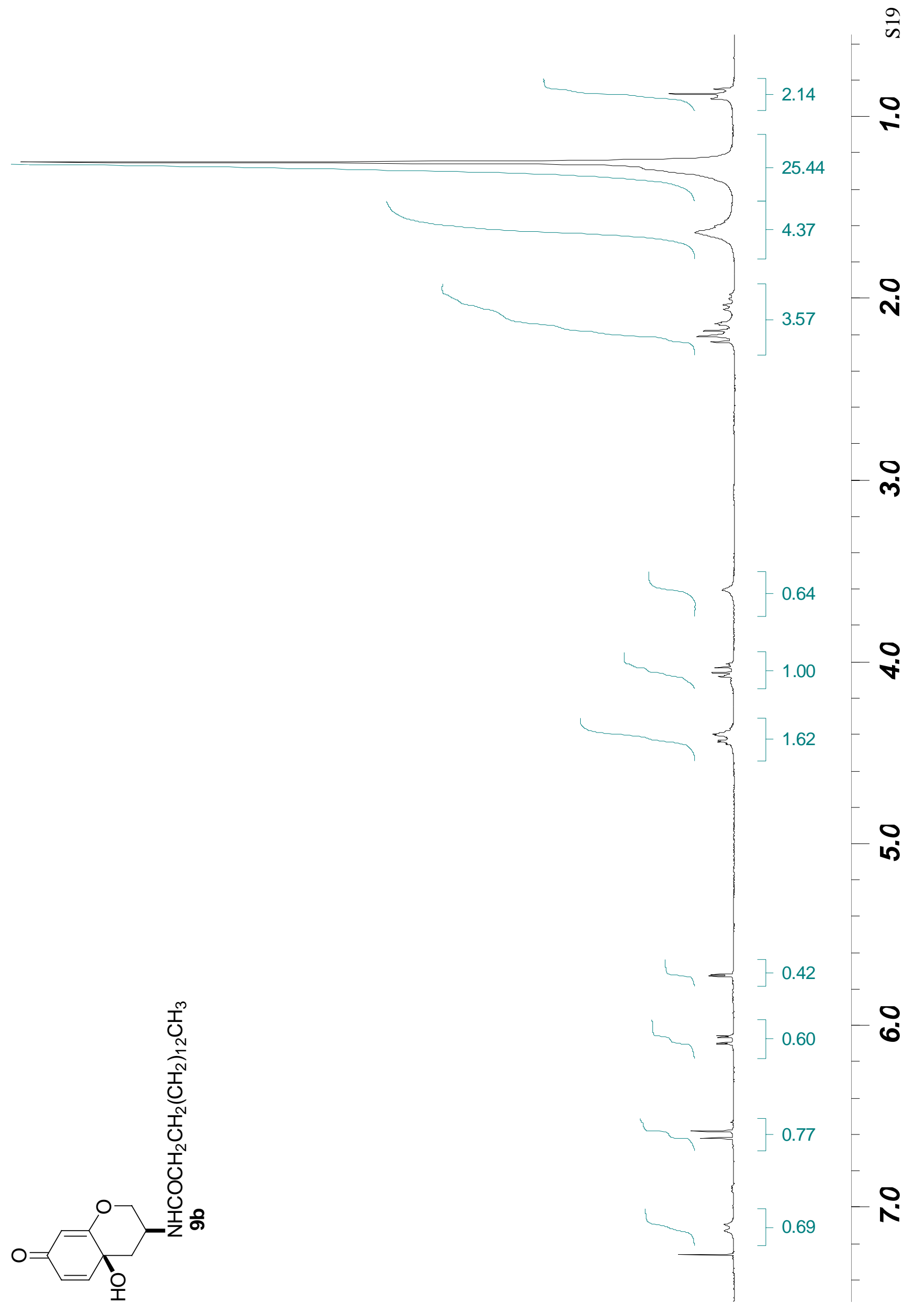




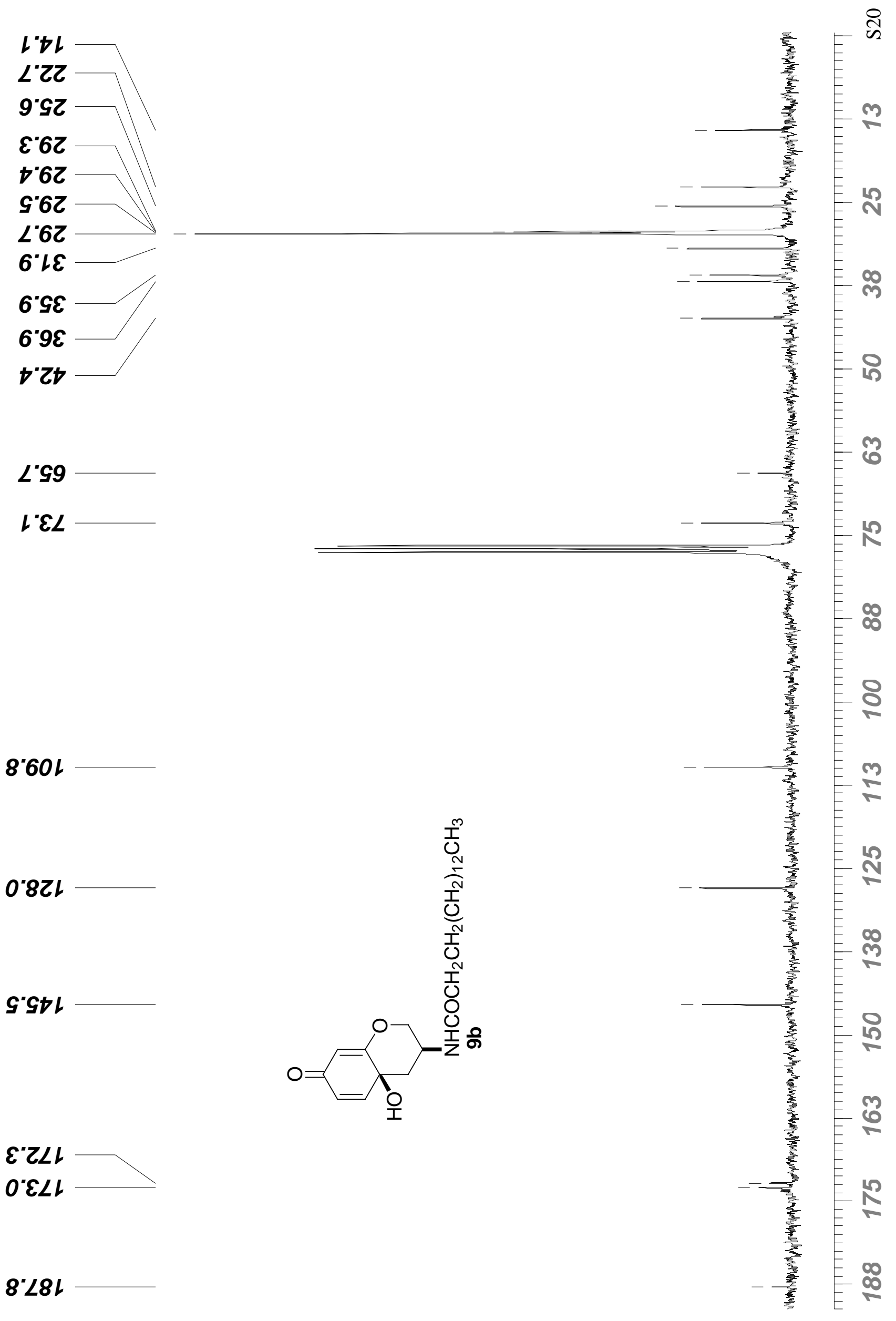




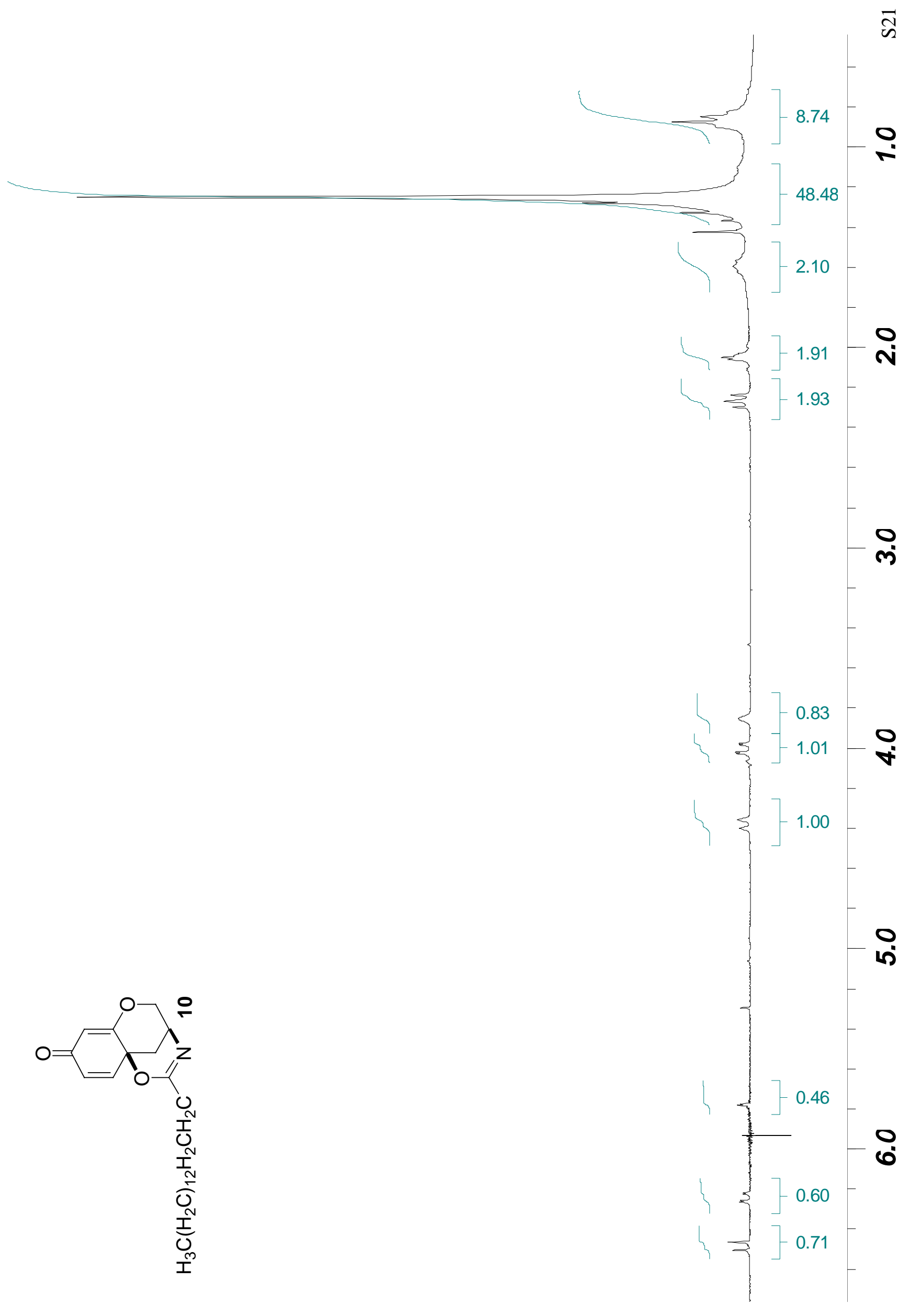




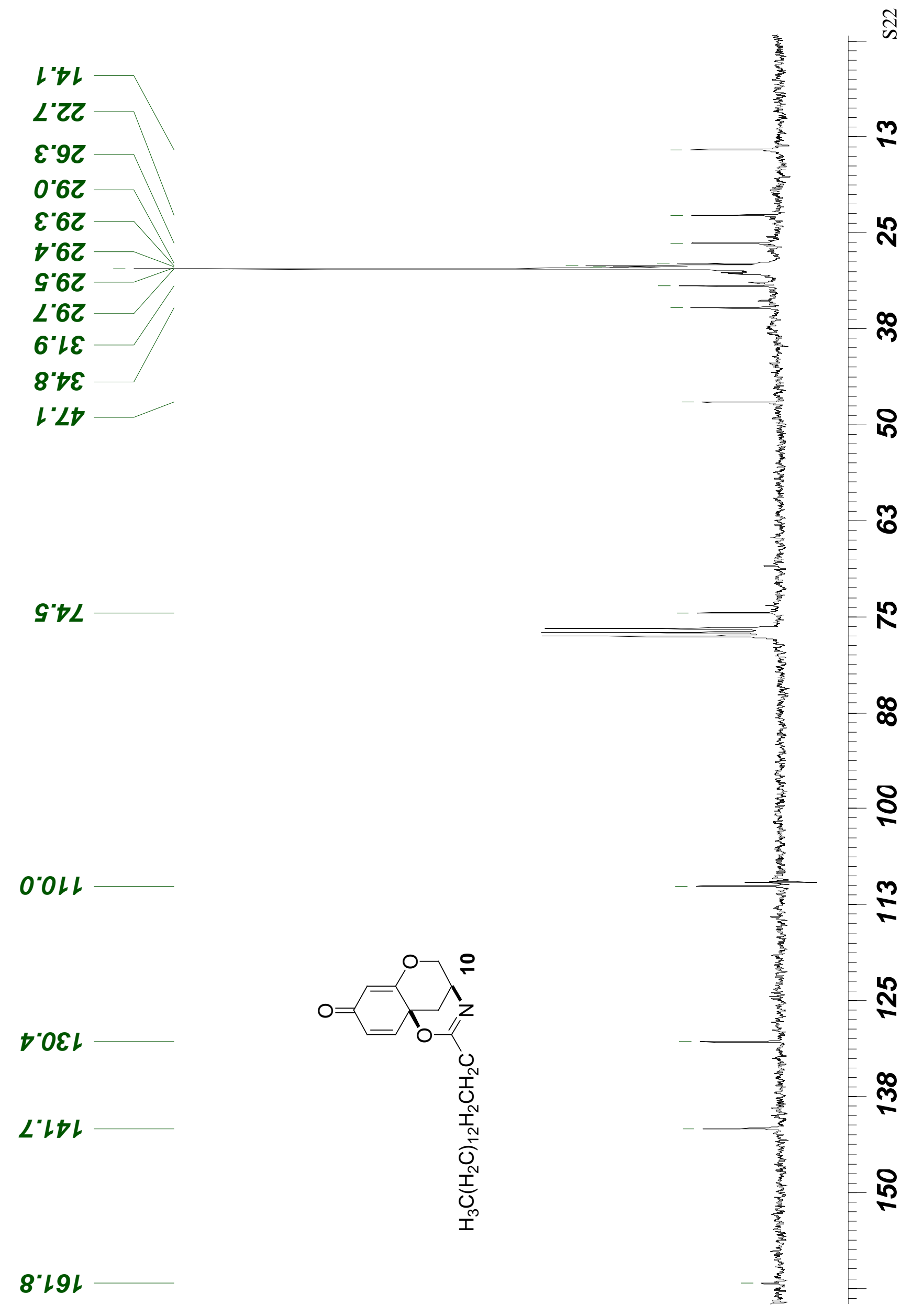


กิ
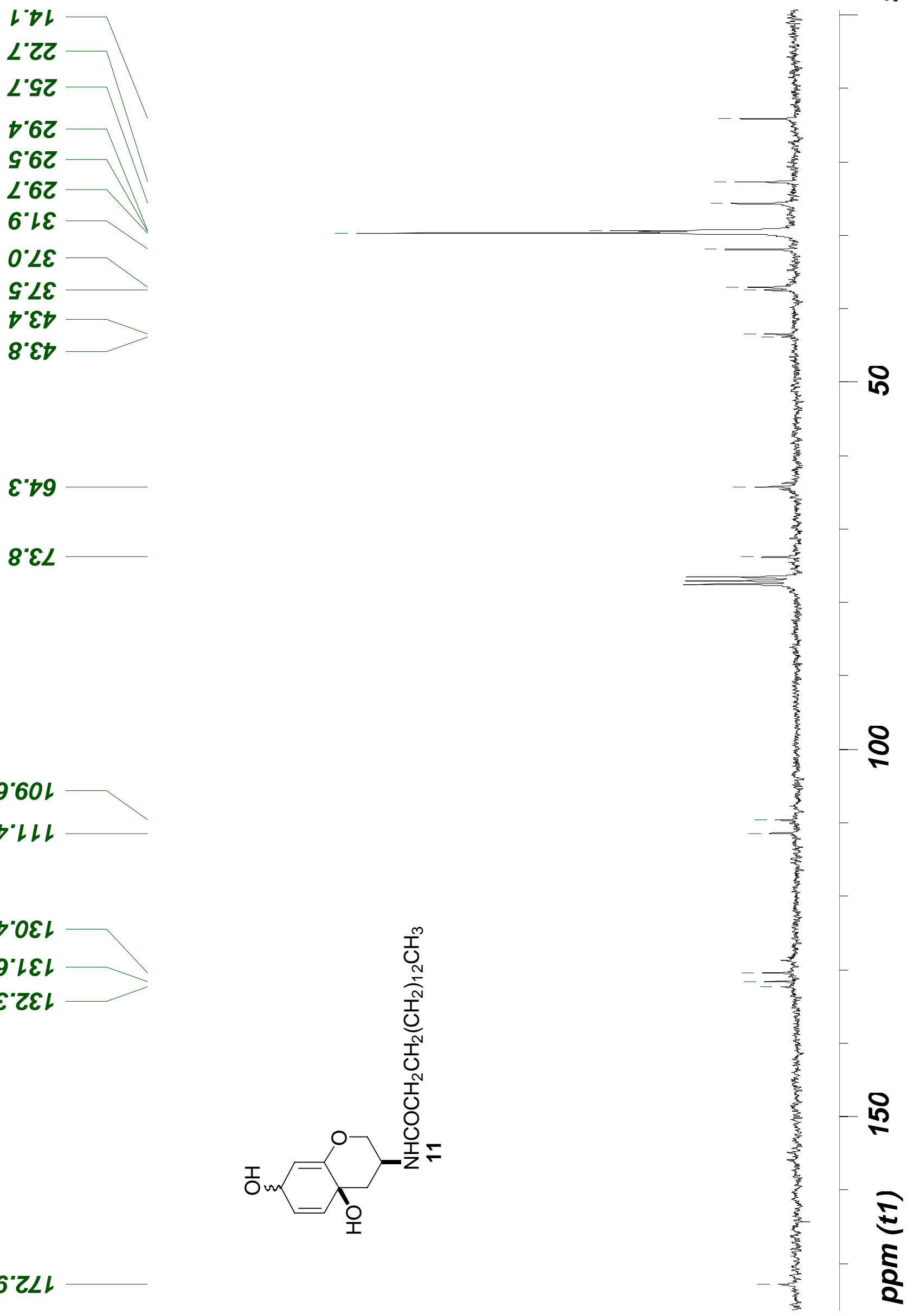


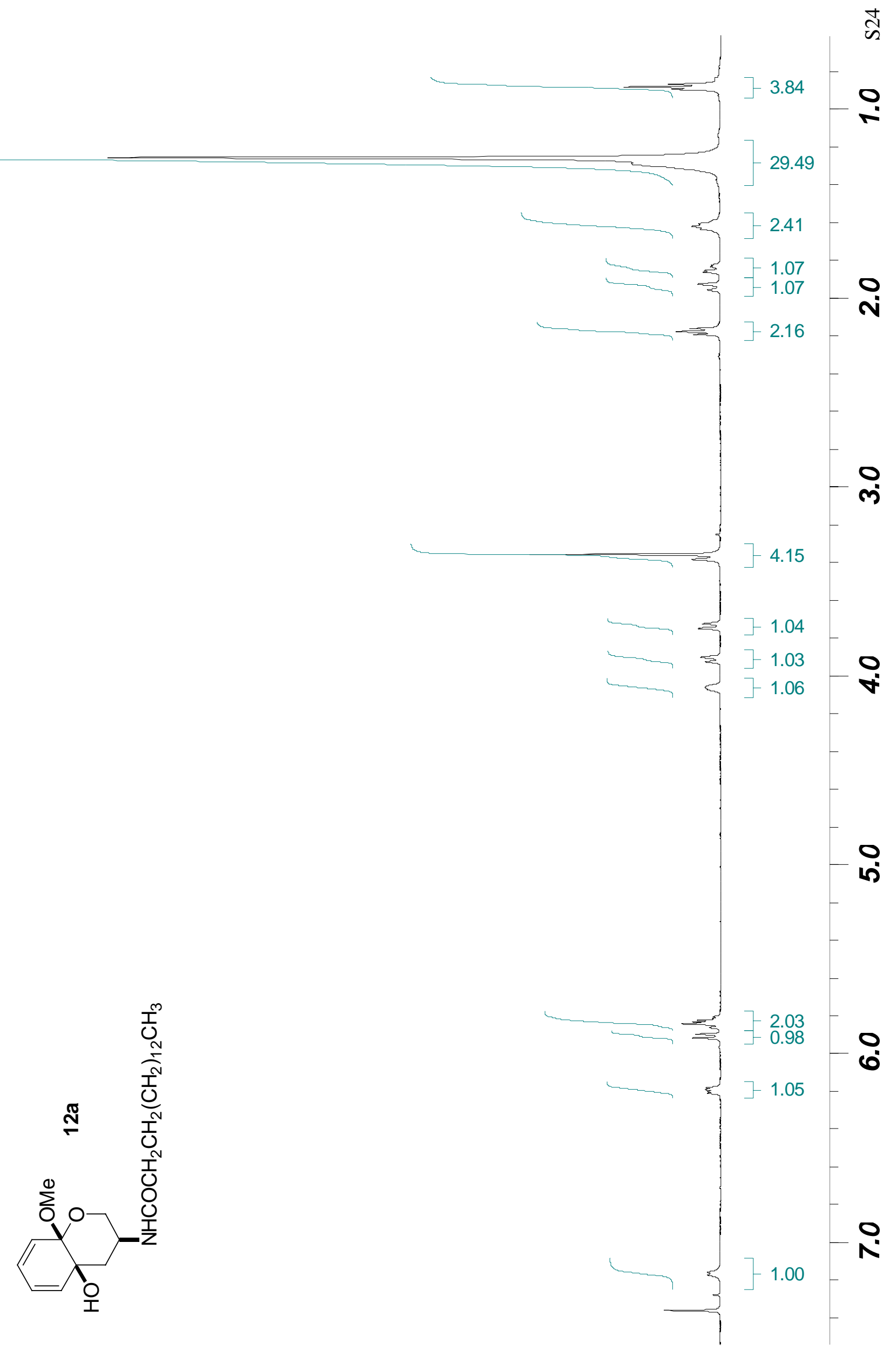




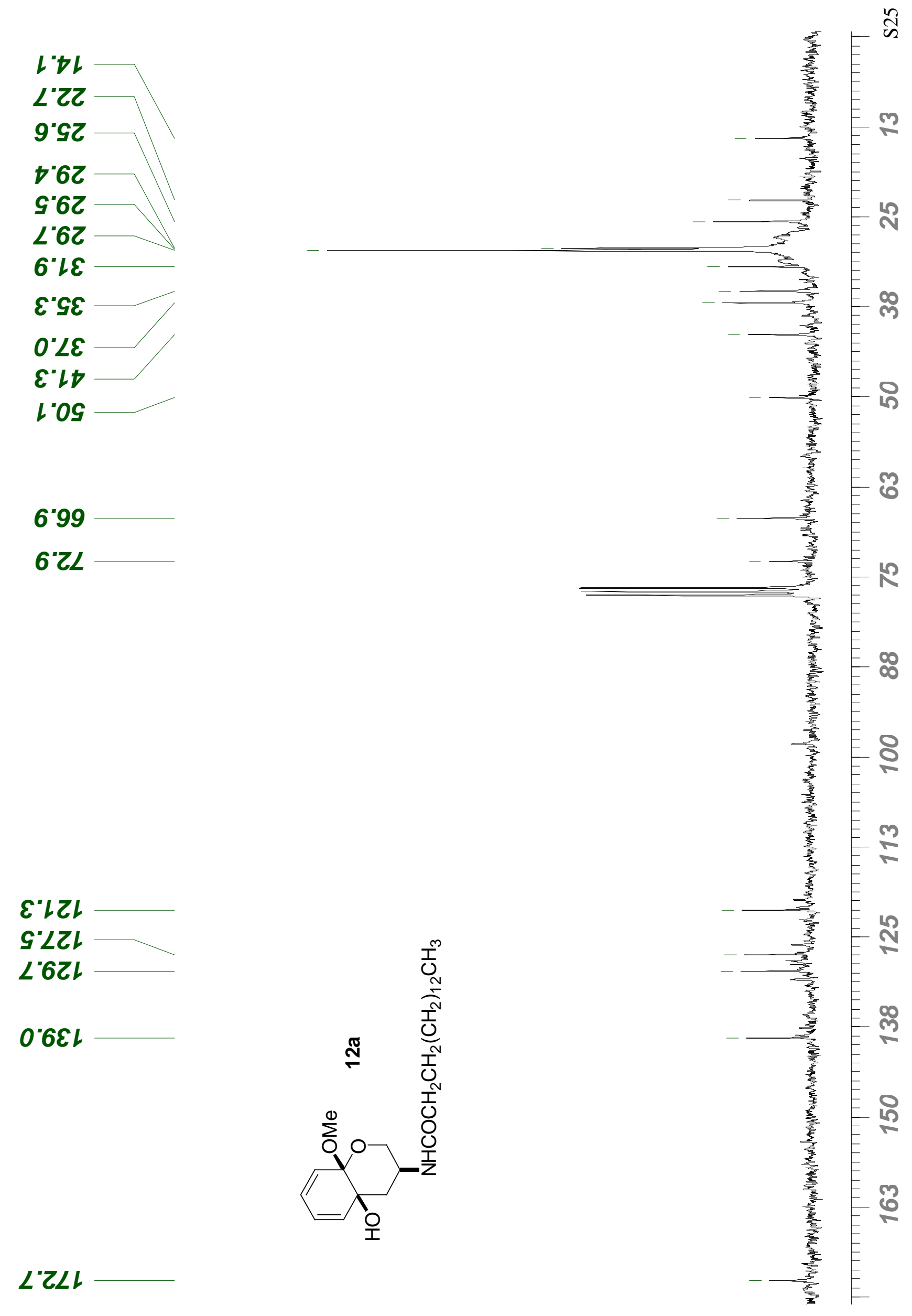




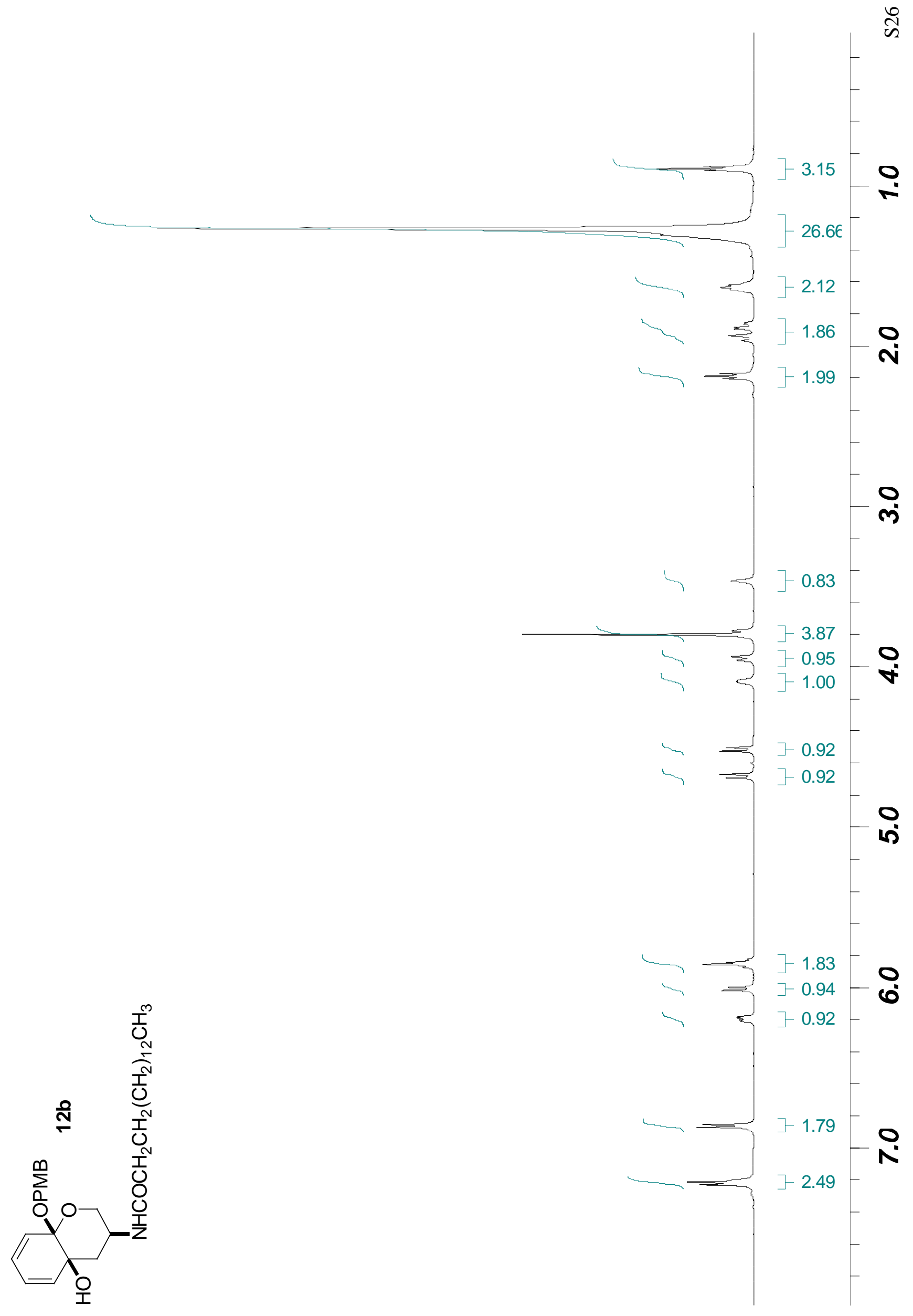




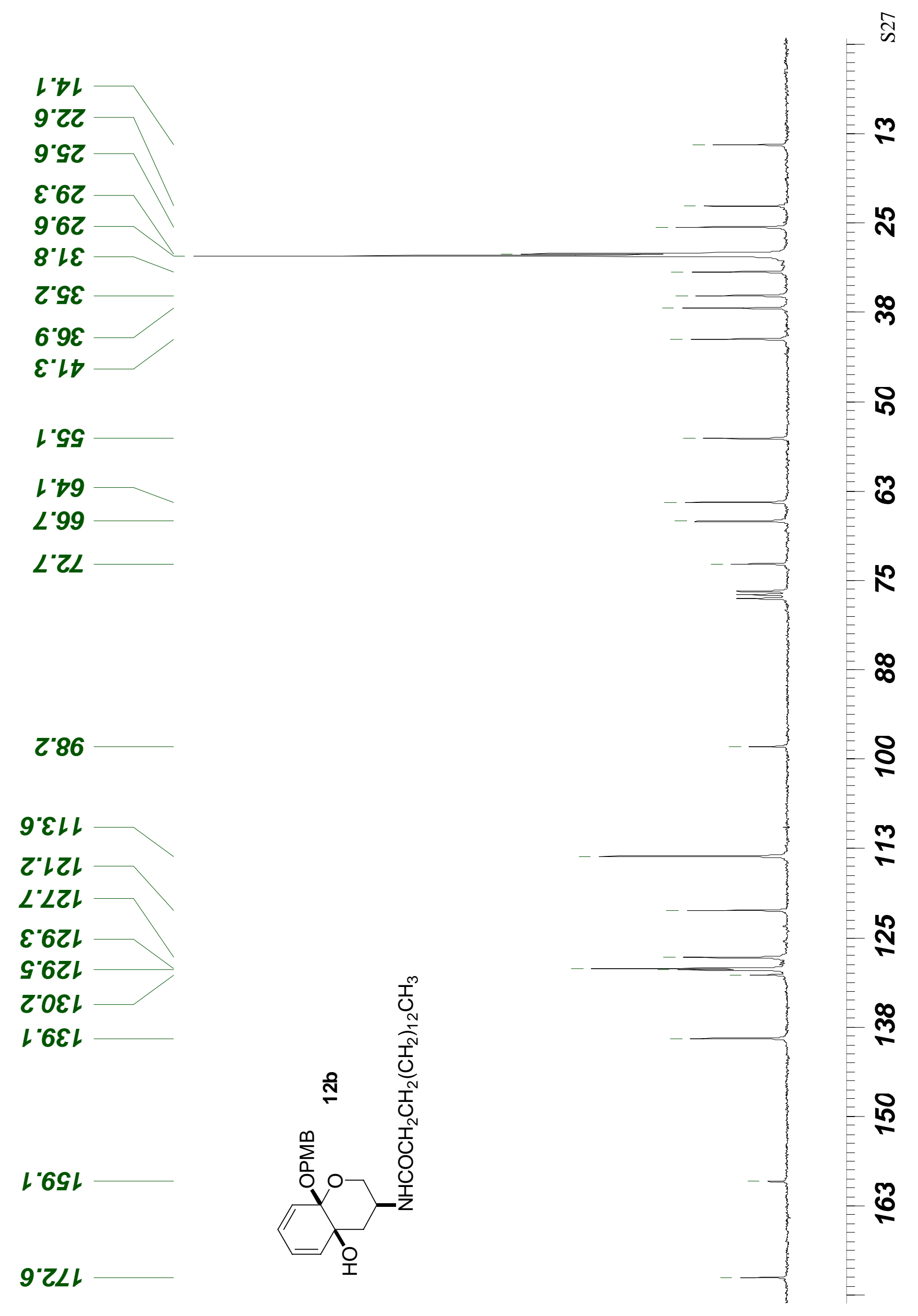




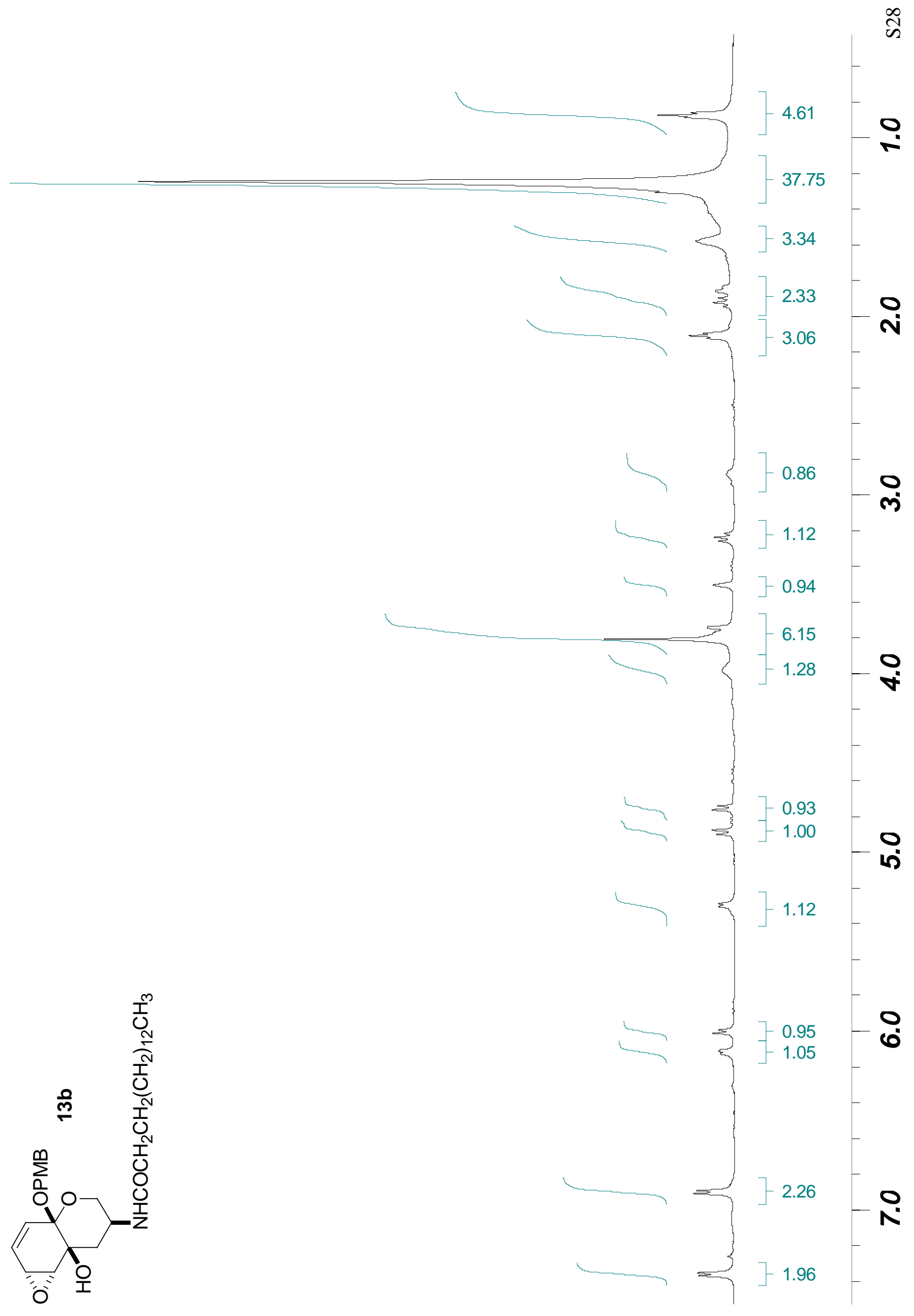




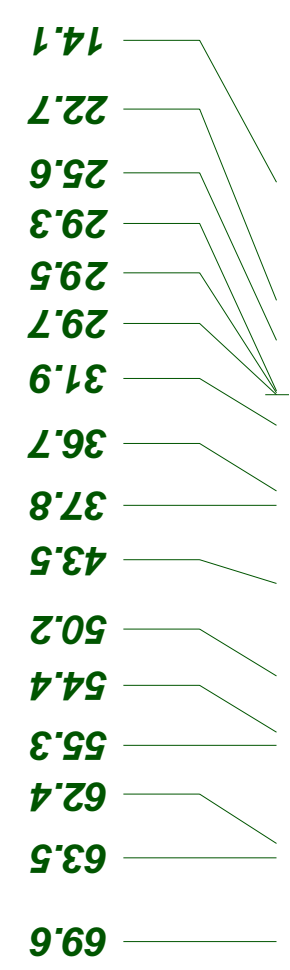

$\varepsilon^{* 96}$

6'ELL

t'SZL

9'8ZL

S'6ZL

ᄂ'0عเ

Z"0tl

Z'6SL

6'ZLL
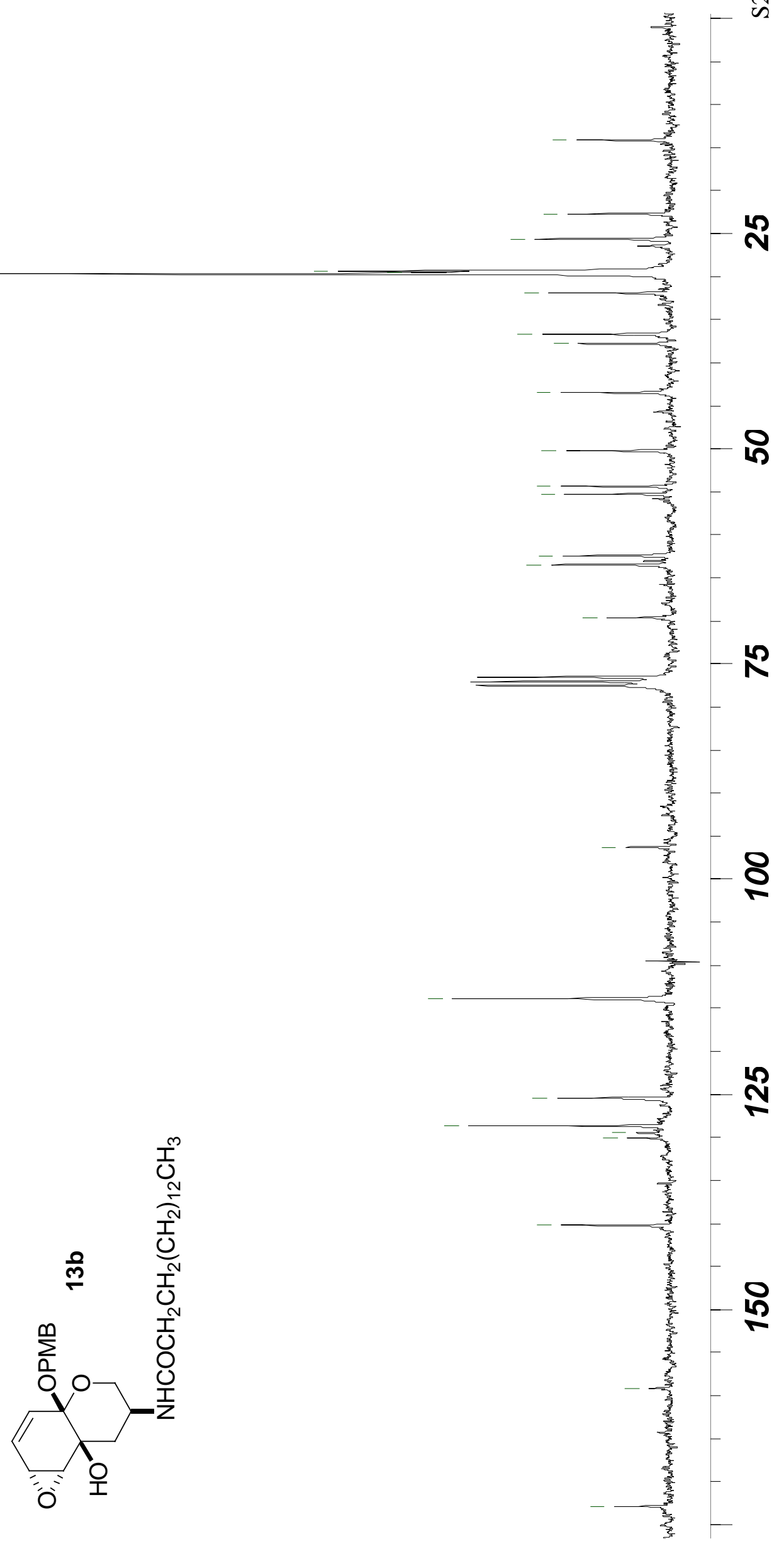


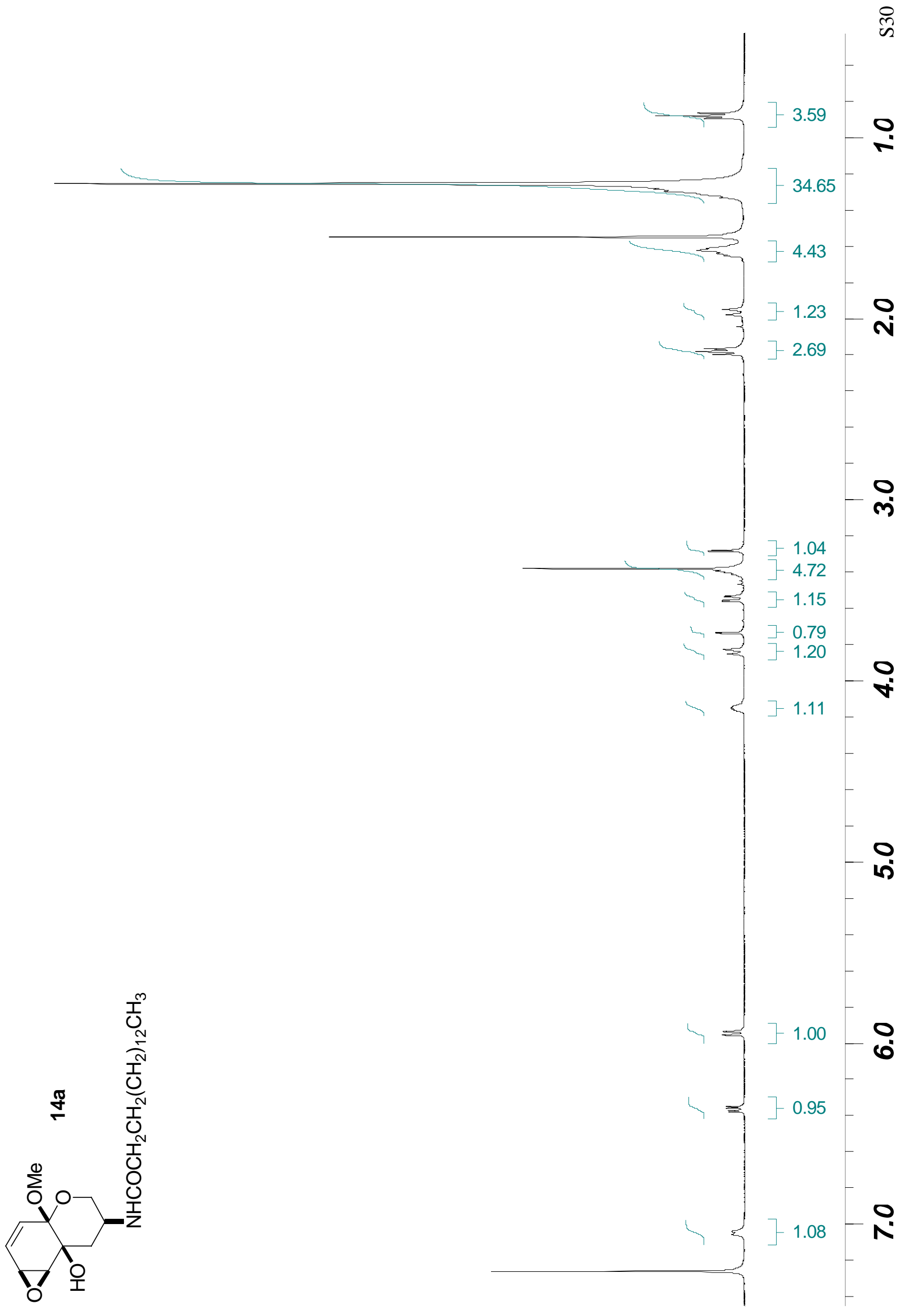




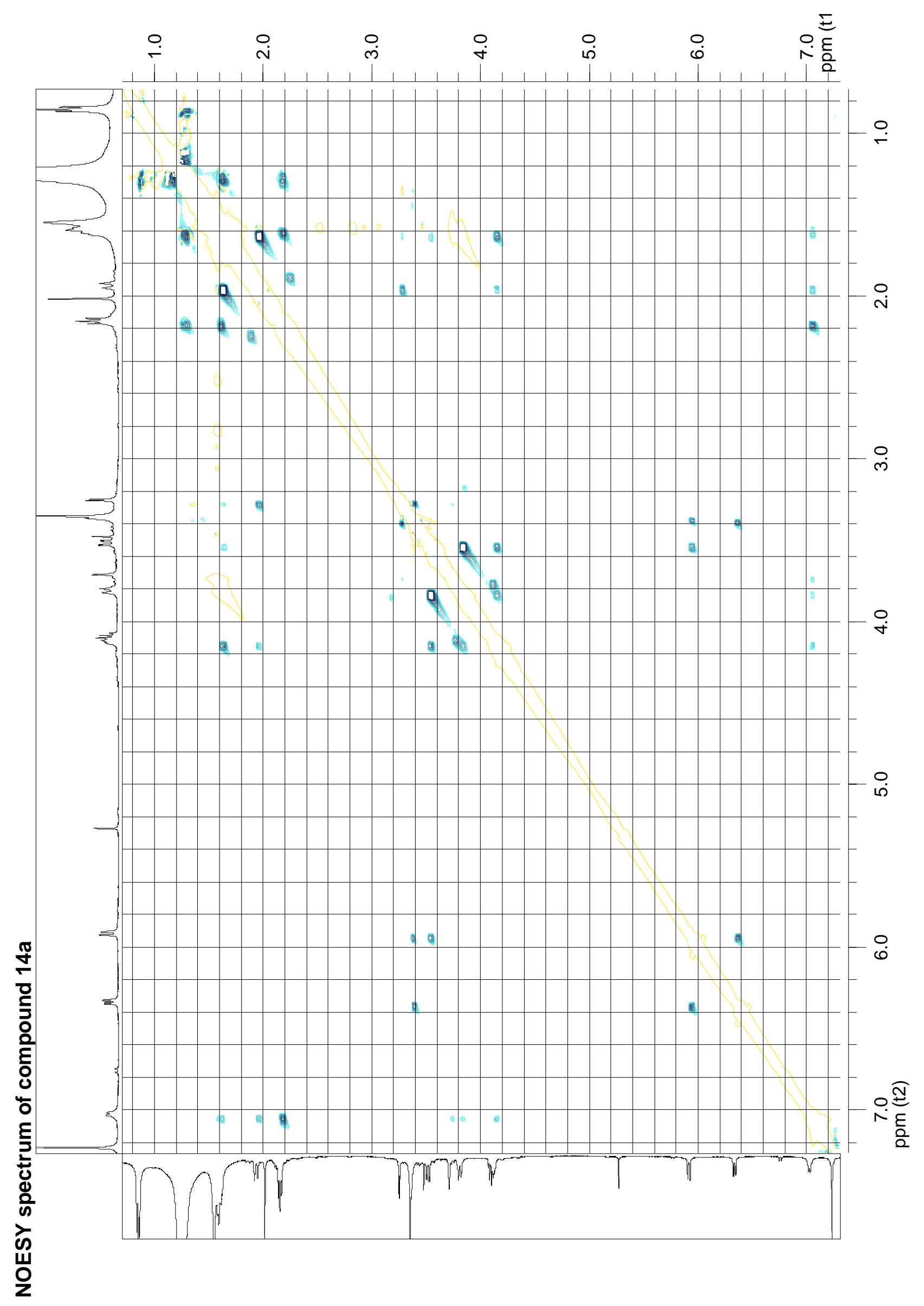




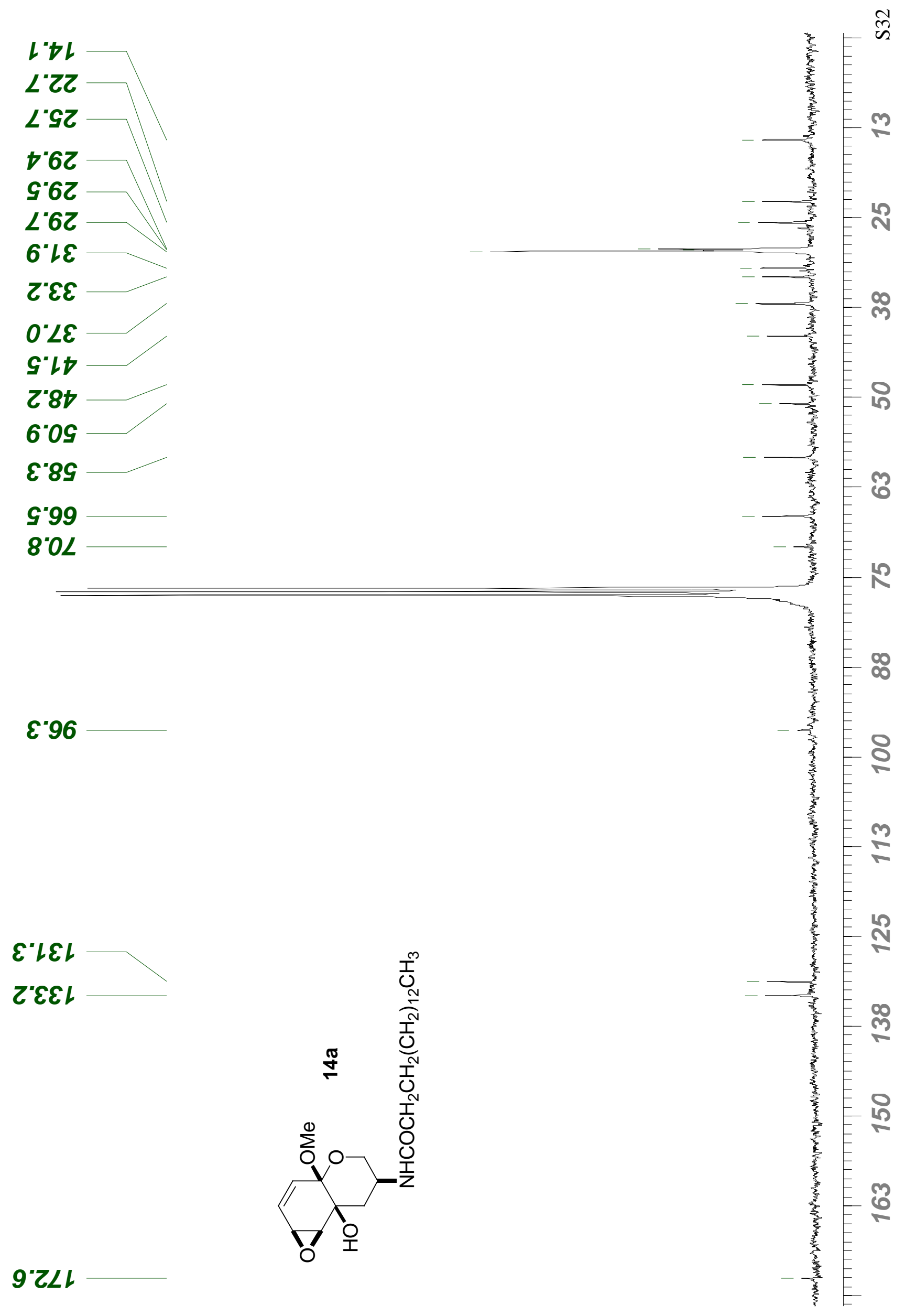




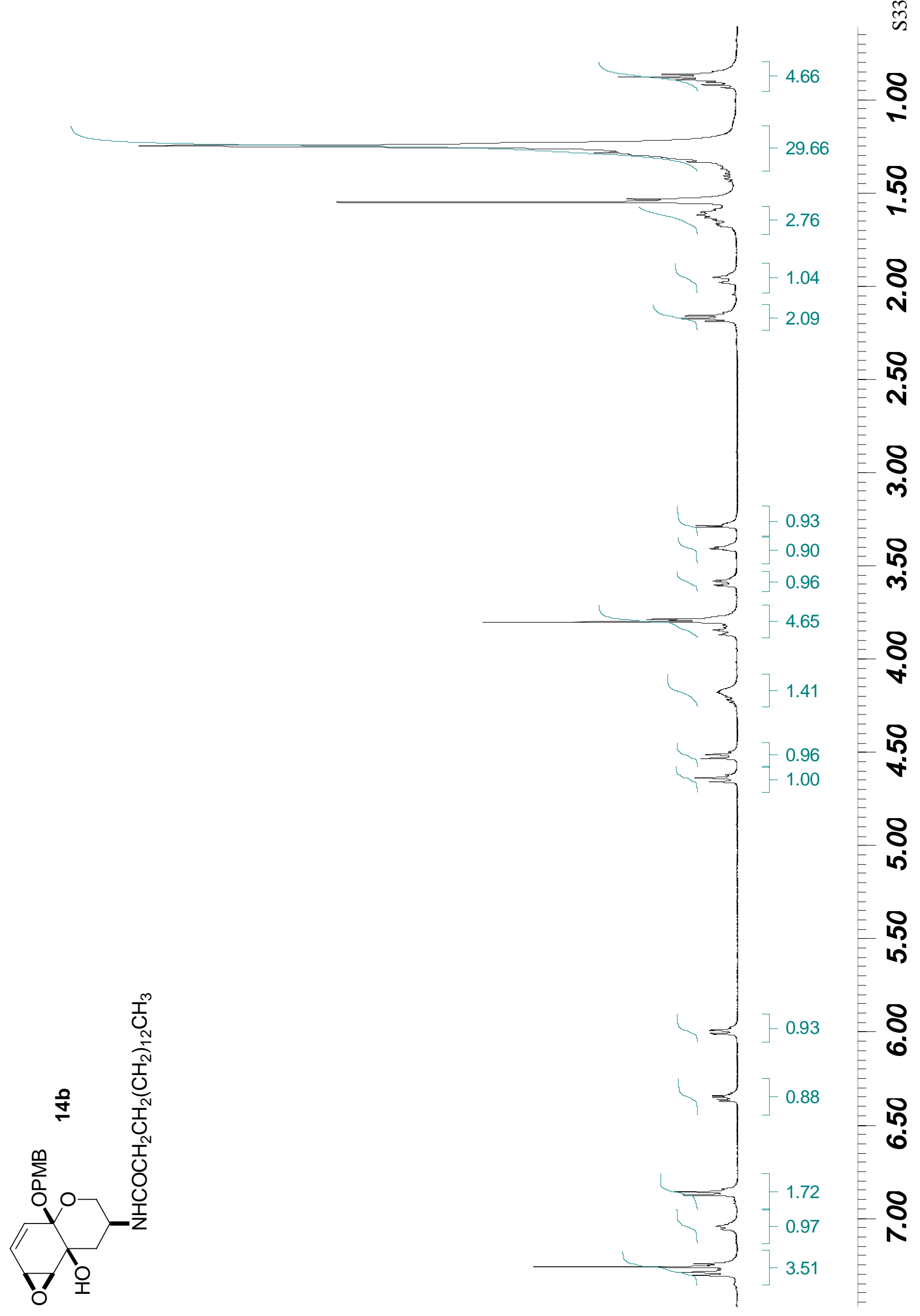




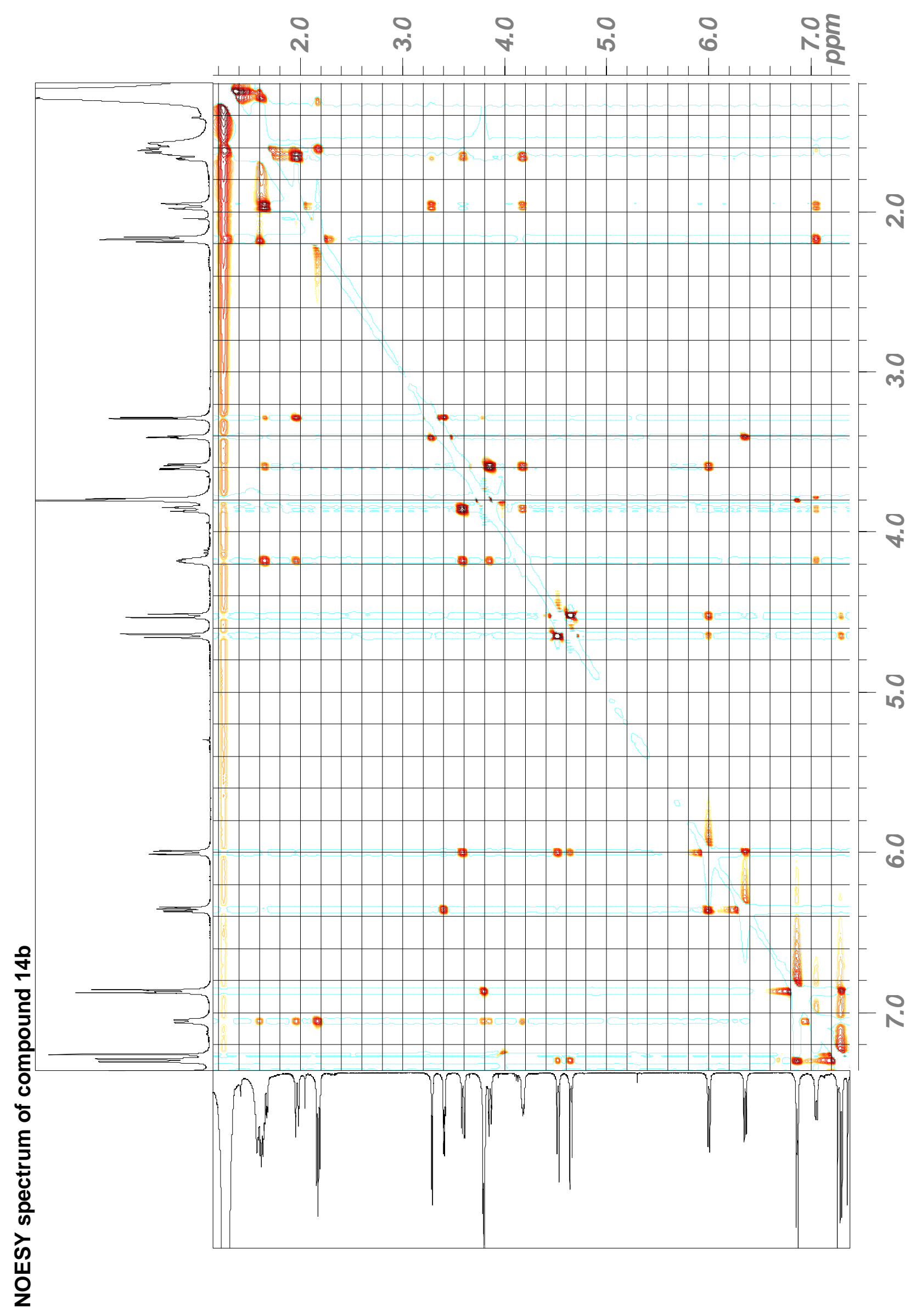




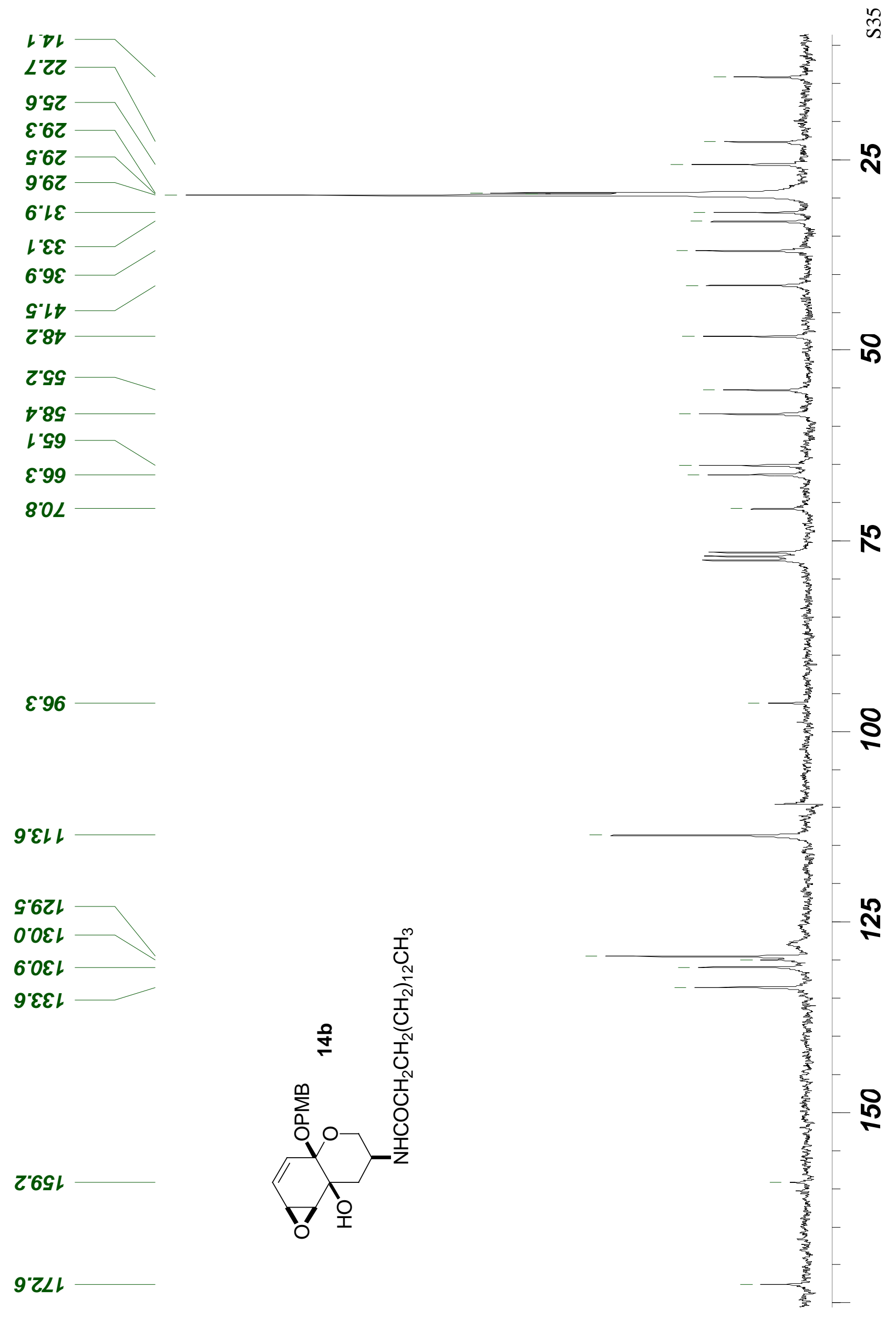




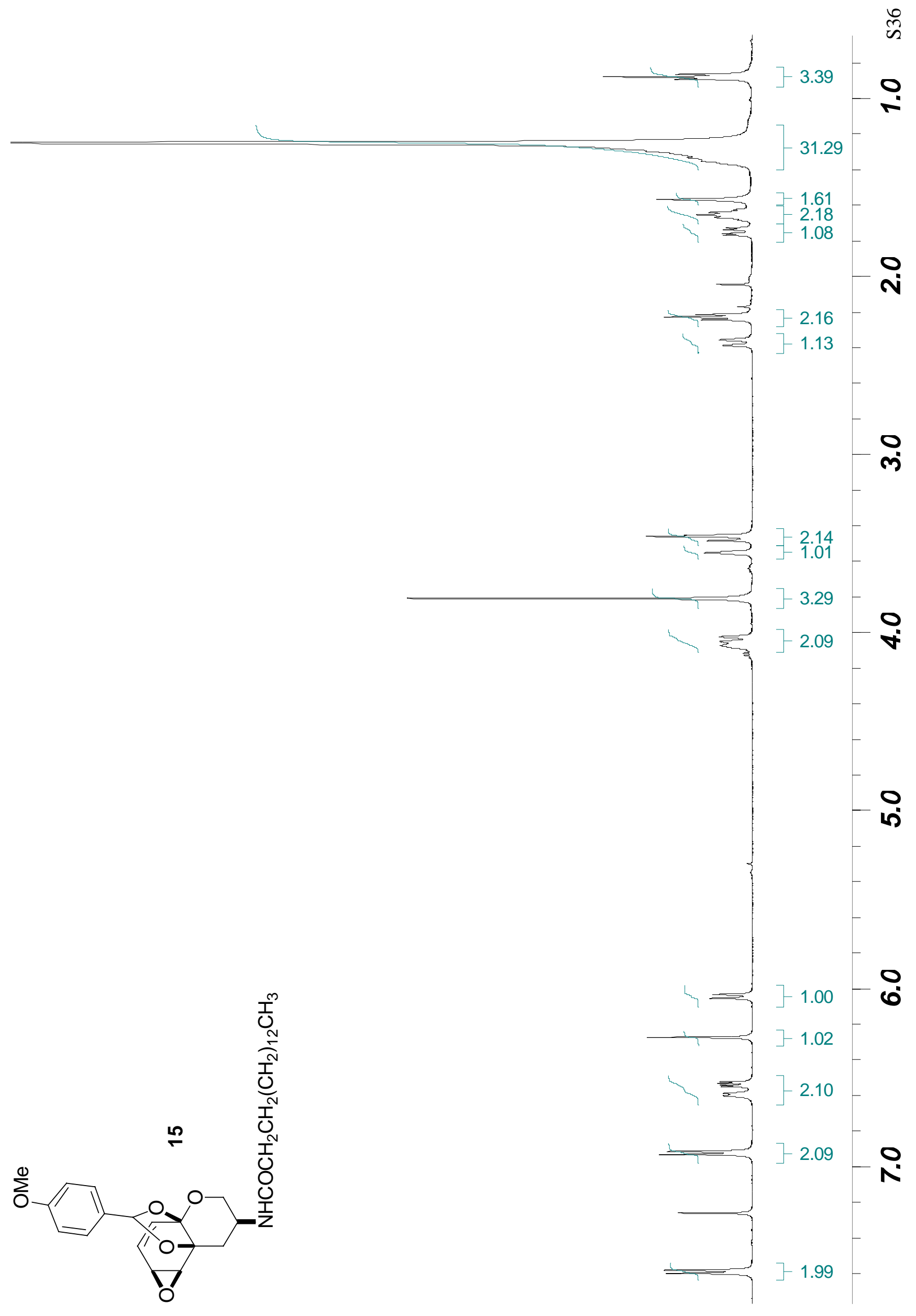




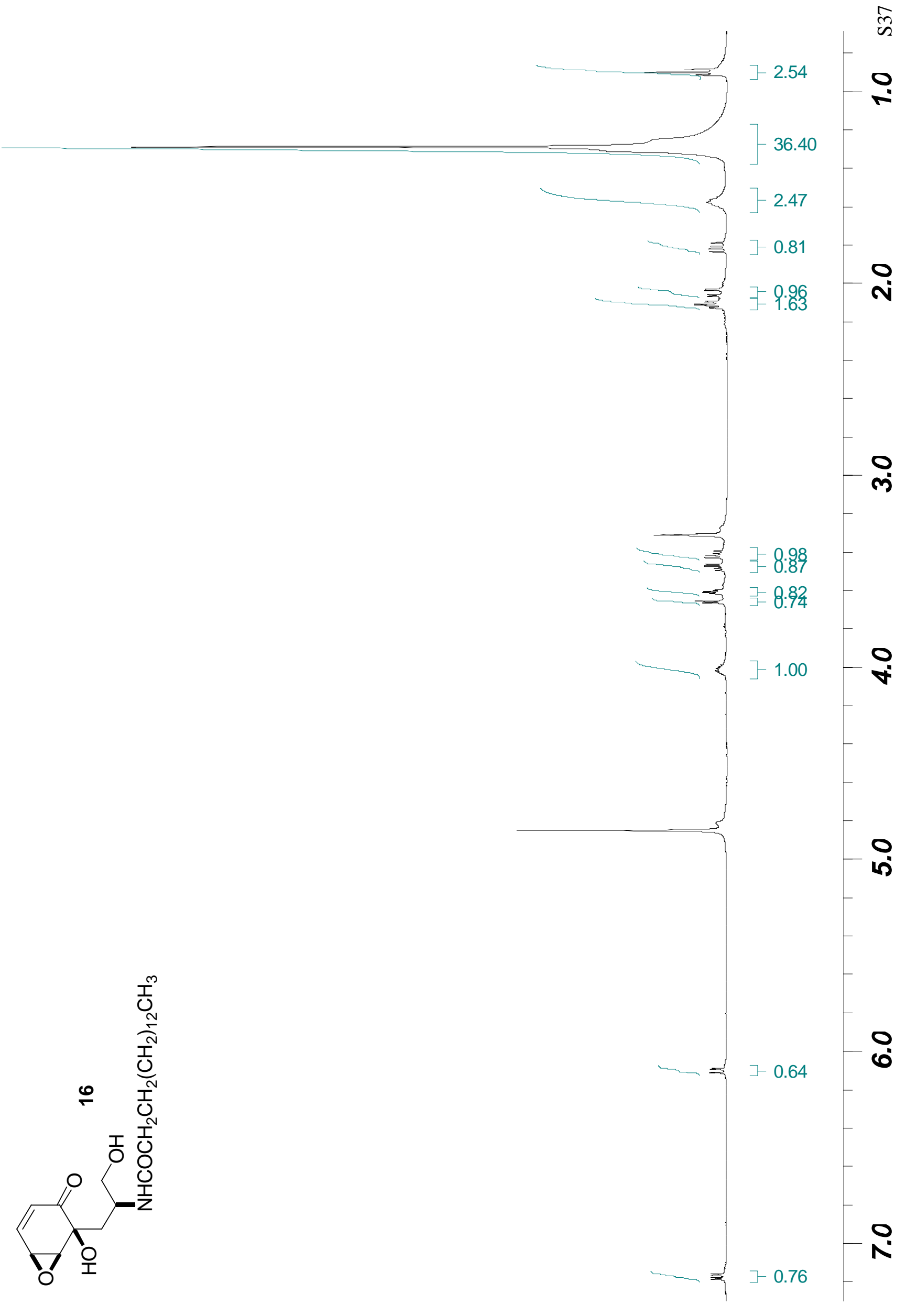




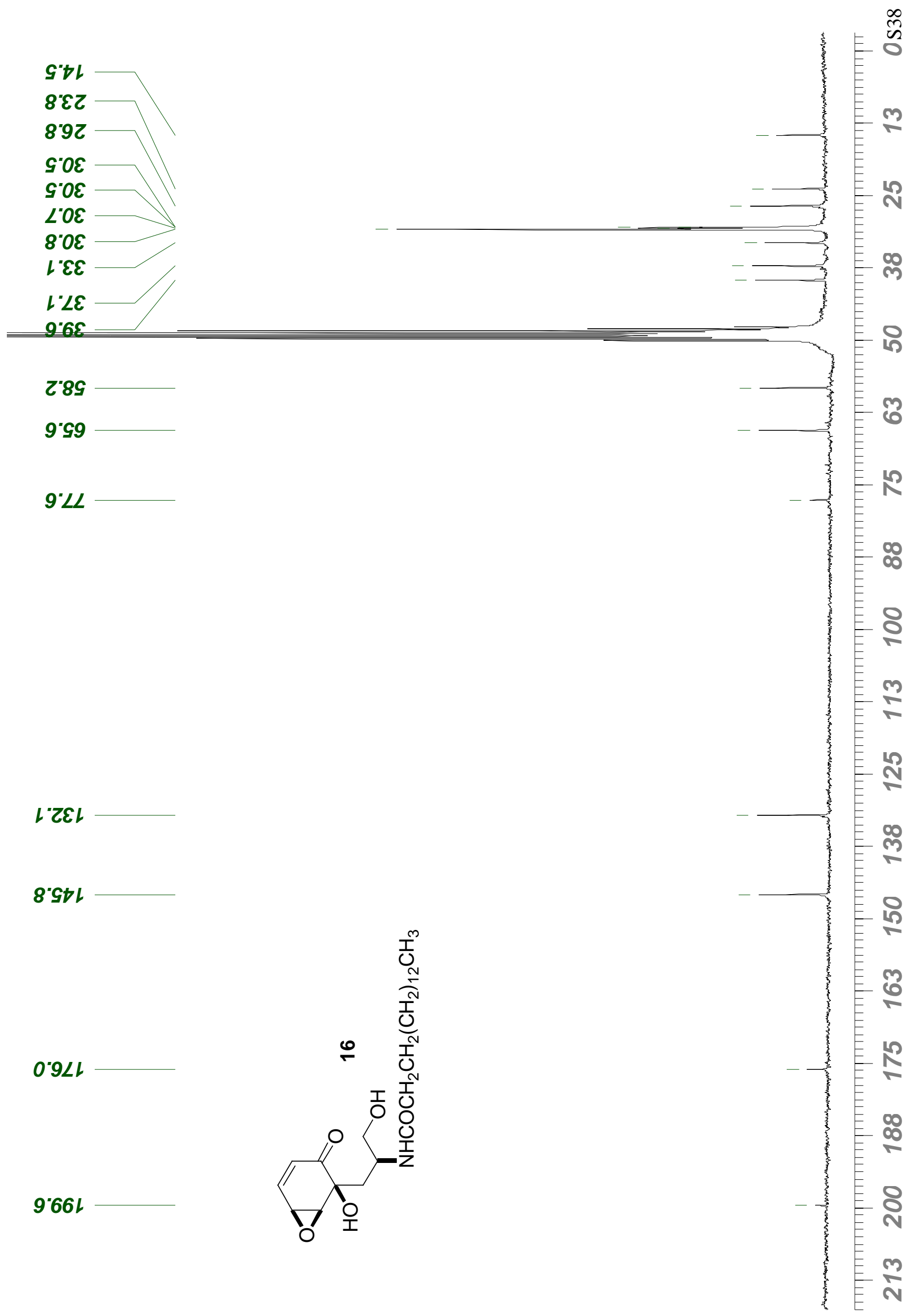




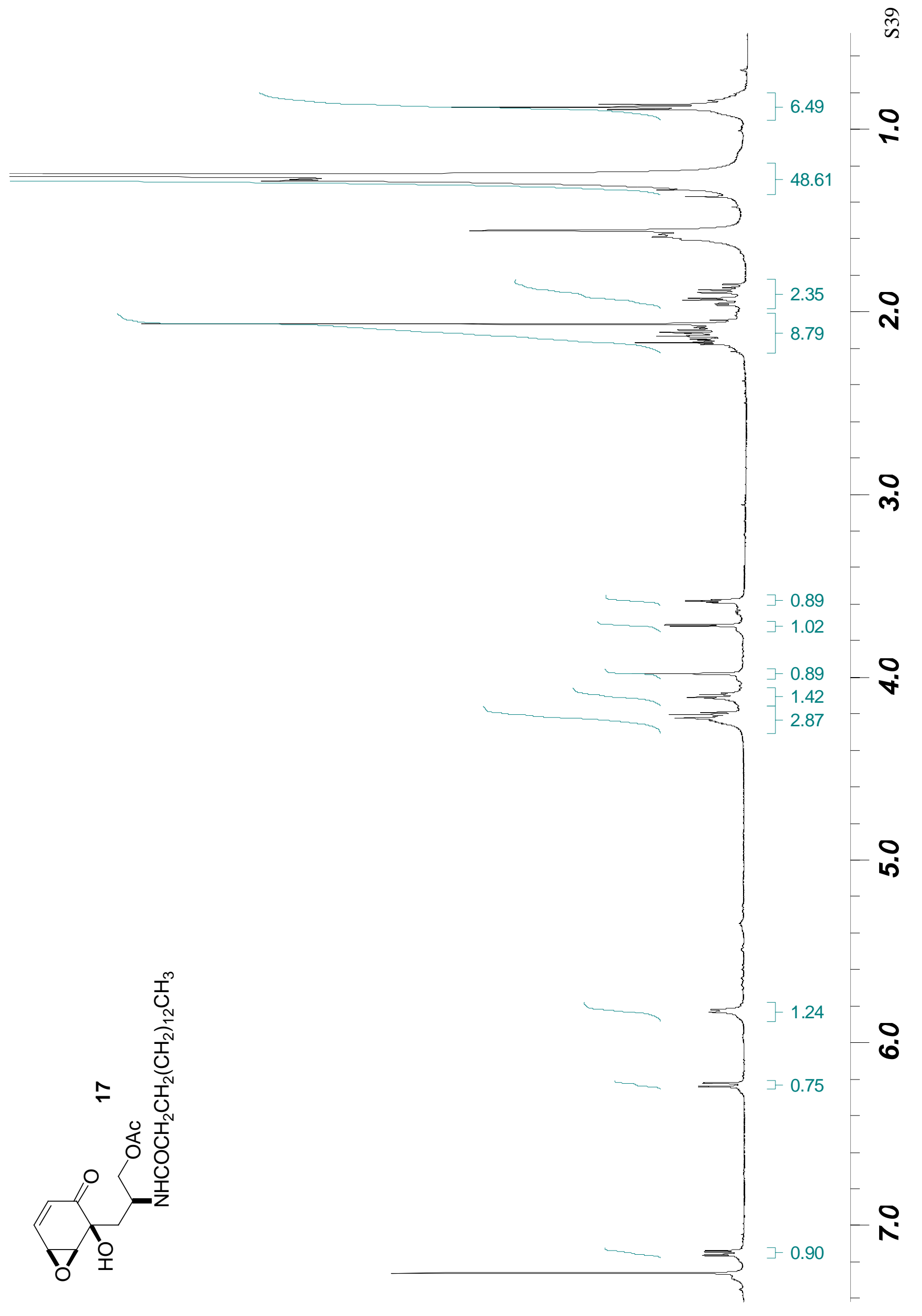

